\title{
Canagliflozin Alleviates Diabetic Cardiovascular Disease via Lipid Lowering, Mitochondrial Homeostasis, and Gut Microbiota Regulation
}

\section{Xueliang Wang}

Sun Yat-sen University First Affiliated Hospital

\section{Zhe Wang}

Ocean University of China

Di Liu

Ocean University of China

Hao Jiang

Ocean University of China

\section{Chao Cai}

Ocean University of China

\section{Guoyun Li}

Ocean University of China

Guangli Yu ( $\nabla$ glyu@ouc.edu.cn )

Ocean University of China https://orcid.org/0000-0002-6372-9750

\section{Original investigation}

Keywords: Canagliflozin, Mitochondrion, Hypolipidemic, Colonic microbiota, Myocardial protection, Diabetic cardiovascular disease

Posted Date: August 12th, 2021

DOI: https://doi.org/10.21203/rs.3.rs-754308/v1

License: (a) (1) This work is licensed under a Creative Commons Attribution 4.0 International License. Read Full License 


\section{Abstract}

Background: Type 2 diabetes mellitus (T2DM) is associated with cardiovascular disease (CVD). Sodium glucose cotransporter 2 inhibitors (SGLT2i) represent the most effective oral drugs for the treatment of T2DM, and have been shown to improve generalized vascular dysfunction. However, it's effect on improving diabetic CVD and the possible action mechanism are currently unclear.

Methods: T2DM was induced by feeding a high-fat diet (HFD) to male C57BL/6J mice for 24 weeks. Afterthat, HFD-fed mice were treated with Cana $(50 \mathrm{mg} / \mathrm{kg} / \mathrm{d})$ or positive drug metformin $(225 \mathrm{mg} / \mathrm{kg} / \mathrm{d})$ by oral gavage for 6 weeks. Myocardial mitochondrial morphology and cardiovascular abnormalities were assessed by transmission electron microscope and histological staining; lipids profile and oxidative stress state were assessed by corresponding biochemical assay kits and histological staining; myocardial injury was characterized by H\&E staining, TUNEL, and ELISA assays; colonic gut microbiota was assessed by $16 \mathrm{~S}$ rDNA high-throughput sequencing.

Results: Cana reduced the lipid (such as TG, TC, and LDL-C) accumulation in serum, thus decreased atherogenic index of plasma and arteriosclerosis index values. And, Cana reduced the circulating markers of inflammation (such as TNFa, MCP-1, and IL-6). More importantly, Cana improved cardiac mitochondrial homeostasis and relieved oxidative stress. Myocardial injury was alleviated after Cana treatment with decreasing levels of serous CTn I and SCD40L. Thus, cardiovascular abnormality was relieved by elevating the CD31 expression level, and suppressing fibrosis and basement membrane thickening in T2DM mice. Interestingly, Cana administration increased the ratio of Firmicutes/Bacteroidetes and the relative abundance of Olsenella, Alistipes, and Alloprevotella, while decreased the abundance of Proteobacteria, Helicobacter and Mucispirillum at various taxonomic levels.

Conclusions: Cana treatment improved CVD by decreasing the risk of atherosclerosis, maintaining a relatively normal haemogram, and reducing the thickness of vascular basement membrane and myocardial injury. Importantly, Cana treatment significantly elevated myocardial mitochondria homeostasis, thus ameliorated the oxidative stress and inflammatory states. Moreover, Cana subtly altered microbiota composition in T2DM mice with CVD, which contributed to the improvement of CVD. Collectively, the improvements of myocardial mitochondrial and gut microbiota homeostasis, may represent an important mechanism underlying the cardiovascular benefits of Cana treatment.

\section{Background}

Type 2 diabetes mellitus (T2DM) is the most common metabolic disease and the prevalence of T2DM is increasing worldwide at alarming rates [1]. It is well known that T2DM is a metabolic disorder characterized by insulin resistance (IR) and chronic hyperglycemia with excessive insulin secretion. Individuals with T2DM are at high risk for macrovascular complications (such as cardiovascular, cerebrovascular, and renal comorbidities), owing to hyperglycaemia and IR [2]. T2DM is associated with cardiovascular disease (CVD) characterized by increases in large artery stiffness, endothelial dysfunction, 
and vascular smooth muscle dysfunction [3]. Risk factors of CVD include hyperglycemia, dyslipidemia, IR, obesity, hypertension, non-alcoholic fatty liver disease and so on $[4,5]$. Among the myriad health consequences of T2DM, CVD accounts for more than two thirds of all deaths [6]. Although there is a strong correlation between the magnitude of hyperglycemia and CVD event rates among people with T2DM $[7,8]$, there remains considerable uncertainty as to whether current anti-diabetic medications reduce CVD risk $[9,10]$.

Inflammation, oxidative stress, and dyslipidemia are key contributing factors to the development of diabetic CVD [11]. It was demonstrated that high fat diet (HFD)-induced elevated cholesterol and low density lipoprotein-cholesterol (LDL-C) resulted in both ischemic heart disease with or without inflammation [12]. Oxidative stress and inflammation are closely associated with the pathogenesis of diabetic CVD [13]. Reactive oxygen species (ROS) is increased in patients with T2DM and animal models of T2DM, and overproduction of ROS is associated with hyperglycemia and metabolic disorder related disease [14]. Moreover, mitochondria are the most important sources of ROS, and mitochondrial dysfunction increases electron leak and ROS production. It is crucial to comprehensively understand the pathophysiological mechanisms mediated by oxidative stress in the prevention and treatment of diabetic CVD [15]. Hyperlipidemia is an elevation of lipids in the bloodstream [16], which is a major cause of atherosclerosis and atherosclerosis-associated conditions such as coronary heart disease and peripheral vascular disease [17]. Moreover, the lipid-glucose index was positively associated with a higher prevalence of CVD, and could be used as a marker for atherosclerosis [18].

Sodium-glucose cotransporter 2 inhibitors (SGLT2i) were recently introduced to treat T2DM [19]. In metaanalyses of clinical trials in T2DM, three types of SGLT2i, including Canagliflozin (Cana), dapagliflozin (Dapa) and empagliflozin (Empa) could reduce fasting plasma glucose and HbA1c [20-22]. Moreover, SGLT2i have been shown to conduct cardiovascular benefits and reduce the related overall mortality [23]. Early modeling data predicted that, unlike other classes of antidiabetic medications, SGLT2i may have significant beneficial effects on CVD [24, 25]. It has been verified that Empa can improve glycemic parameters, promote lipid metabolism and reduces systolic blood pressure [26], thus favoring a cardioprotective property of the SGLT2i $[27,28]$. It demonstrated that Dapa significantly lowered arterial stiffness, and improved endothelial and vascular smooth muscle dysfunction, which were accompanied by reductions in hyperglycemia and circulating markers of inflammation [25].

In recent years, associations between T2DM and altered gut microbiome composition have been reported [29]. Moreover, many researches indicate that the gut microbiota has emerged as critical regulators of human physiology and disease processes, including in cardiovascular function in T2DM [30-32]. Various drug have been shown to profoundly alter gut microbiota composition, which in turn altered drug absorption, metabolism, and mediate the physiological effects of T2DM medications [33-35]. Due to this bi-direction relation extends to various medications for CVD and T2DM, there may be tremendous clinical relevance in the interaction between gut microbiota and SGLT2i. For example, it has been verified that Dapa was associated with subtle alterations in microbial richness and diversity in T2DM mice [25]. 
Cana was recently developed as a type of SGLT2i to improve T2DM and related diseases in an insulinindependent manner [36, 37]. It has been reported that only Cana marked activated AMP-activated protein kinase (AMPK), but not Dapa, or Empa, which suggest a potential additional benefit of Cana therapy compared with other SGLT2i [38]. In addition, Cana decreased serum leptin and IL-6, while increased serum adiponectin and tumor necrosis factor a (TNFa) in T2DM patients, which favorably impacted insulin sensitivity and CVD risk [39]. Recently, several clinical studies indicated that the risk of kidney failure and cardiovascular events was lower after Cana treatment in patients with T2DM and kidney disease [40-42]. In addition, Cana significantly altered cecal microbiota composition in renal failure mice and decreased the levels of microbiota-derived uremic toxins in chronic kidney disease [43].

Here, we examined the effects of Cana on attenuating CVD using T2DM mice induced by HFD. Meanwhile, metformin, as a first-line clinical drugs for T2DM, revealed marked improvement in various clinical parameters including a conspicuous reduction in all-cause mortality and CVD events [44]. Thus, metformin was selected as the positive control. With the above background, the purpose of the present study was to examine the effects of Cana on hyperlipemia, systemic inflammation, oxidative stress, IR, myocardial mitochondria, and vascular basement membrane in T2DM mice, and the effects of Cana on the gut microbiota dysbiosis was also characterized.

\section{Methods}

\section{Chemicals and materials}

Cana was purchased from Selleck Chemicals (Selleck Chemicals, Houston, TX, USA); metformin was purchased from Aladdin (Aladdin Bio-Chem Technology Co., Shanghai, China). All other chemicals at analytical grade were purchased from Sigma-Aldrich (Sigma-Aldrich, St. Louis, MO, USA) unless otherwise noted.

\section{Animals and experimental design}

The experimental protocol is shown in Fig. S1. Briefly, specific pathogen free male C57BL/6J mice aged 6 weeks with weight of 20-22 g were obtained from the Charles River Laboratory Animal Technology Co. Ltd (Beijing, China). Mice were allowed to acclimatize the laboratory environment ( $12 \mathrm{~h} \mathrm{light/dark} \mathrm{cycle} \mathrm{at}$ $23-25^{\circ} \mathrm{C}$ and $30-60 \%$ relative humidity) for one week before the beginning of the experiment. All mice were free accessed to food and water. The mice were randomly divided into four groups ( $n=10$ per group): normal-chow diet (NCD) fed group (Control group) (D12450B, containing 10\% kcal from fat, 3.85 total $\mathrm{kcal} / \mathrm{g}$, Research Diets Inc., New Brunswick, NJ, USA), HFD fed group (Model group) (D12492, containing $60 \%$ kcal fromfat, 5.24 total kcal/g, Research Diets Inc., New Brunswick, NJ, USA), HFD plus metformin group (Metf group), and HFD plus Cana group (Cana group). Throughout the establishment of T2DM mice model period of 24 weeks, most individuals with HFD feeding appeared to be significantly hyperglycemic compared with the NCD feeding mice, while a few mice maintained a 
relatively normal blood glucose level. Thus, we randomly selected six mice with hyperglycemia for the following treatment trail. The sample size of each group was calculated by G*Power software. In a oneway ANOVA analysis with an effect size of $f=0.7, a$ of 0.05 , power level of $80 \%$ and group number of 4 , it was calculated that a number of 6 mice per group would be sufficient to reach statistical significance. After that, the Metf and the Cana groups were gavaged with metformin $(200 \mathrm{mg} / \mathrm{kg}$ dissolve in saline solution containing $0.5 \%$ carboxymethyl cellulose and $0.025 \%$ Tween-20) or Cana (50 mg/ $\mathrm{kg}$ dissolve in saline solution containing $0.5 \%$ carboxymethyl cellulose and $0.025 \%$ Tween-20) once a day for six more weeks, while the Control group and the Model group were given equal amounts of solvent as a control vehicle. And, body weight was recorded weekly. At the end of the experiment, mice were fasted for 12 $\mathrm{h}$ and were euthanized by intraperitoneal injection of $1 \%$ pentobarbital sodium $(100 \mathrm{mg} / \mathrm{kg})$ [45-47].

\section{Automatic haematological analysis}

Blood samples were collected in $1.5 \mathrm{~mL} \mathrm{~K}{ }_{3}$ EDTA-containing tubes (Sarstedt, Nümbrecht, Germany) via eyeball method and stored at $4^{\circ} \mathrm{C}$. Samples were analyzed using an automated veterinary hematology analyzer Mindray BC 2800VET (Mindray, Shenzhen, China).

\section{Tissue sampling and histological study}

Cardiac tissues were collected, then partial cardiac tissues were immediately washed with PBS and then fixed in a $4 \%$ paraformaldehyde solution or $2.5 \%$ glutaraldehyde for $48 \mathrm{~h}$, while frozen tissues were used for Oil Red 0 staining. Tissues were then dehydrated by ethanol and embedded in paraffin wax. Embedded tissues were processed as previously described and cut into $4 \mu \mathrm{m}$-thick sections [48]. The tissue sections were analyzed by hematoxylin and eosin (H\&E) staining, Oil Red 0 staining, or Masson trichrome staining as previously described [49-51].

\section{Biochemical assays}

Fasting blood glucose (FBG) was measured by using a standard glucometer (Johnson \& Johnson, New Brunswick, NJ, USA) after fasting for $12 \mathrm{~h}$. Oral glucose tolerance test (OGTT) and intraperitoneal insulin tolerance test (IPITT) were conducted as previously described and blood glucose was assayed using a standard glucometer $[52,53]$. The increment of blood glucose following the glucose loading was expressed in terms of area under the curve (AUC), using the trapezoidal rule. Blood was collected with a $1.5 \mathrm{~mL}$ EDTA-free centrifugal tube and immediately centrifuged at 2,000 rpm for $10 \mathrm{~min}$ at $4^{\circ} \mathrm{C}$ to obtain serum for serological test. Serum amounts of fasting insulin and hemoglobin A1c (HbA1c) were determined using a Mouse Insulin ELISA Kit and an HbA1c ELISA Kit (Omnimabs, Alhambra, CA, USA) according to the manufacturer's instruction, respectively. Homeostasis model assessment-insulin resistance (HOMA-IR) was calculated as fasting insulin $\left(\mathrm{mU} \mathrm{L}^{-1}\right) \times$ fasting glucose $(\mathrm{mM}) / 22.5$. Serum total cholesterol (TC), triglyceride (TG), low-density lipoprotein cholesterol (LDL-C), and high-density 
lipoprotein cholesterol (HDL-C) levels were detected by standard assay kits (Nanjing Jiancheng Bioengineering Institute, Nanjing, China) according to manufacturer's instructions. Serum amounts of monocyte chemotactic protein 1 (MCP-1), interleukin-6 (IL-6), and TNFa were determined using ELISA kits (R\&D systems, Minneapolis, MN, USA). Cardiac troponin I (cTn I) and soluble cluster of differentiation 40 ligand (SCD40L) contents in serum were determined using the corresponding ELISA kits (Wuhan Jiyinmei Biotechnology Co. Ltd., Wuhan, China). Cardiac tissues $(200 \mathrm{mg})$ were homogenized in cold phosphate buffer ( $\mathrm{pH} 7.4$ ), then centrifuged at $10,000 \mathrm{~g}$ for $20 \mathrm{~min}$ to collect the supernatant. The concentrations of superoxide dismutase (SOD), glutathione (GSH), catalase (CAT), and malondialdehyde (MDA) in cardiac tissue were determined according to the product manual (Nanjing Jiancheng Bioengineering Institute, Nanjing, China). In addition, ROS content in cardiac tissue was detected by the fluorescent dye dihydroethidium (DHE) as previously described with minor modifications [54]. For in situ detection of ROS, OCT-embedded tissue was cut into $4 \mu \mathrm{m}$-thick sections. Tissue sections were then incubated with $5 \mu \mathrm{M}$ of DHE for $30 \mathrm{~min}$ at $37^{\circ} \mathrm{C}$ in the dark. After the application of a coverslip, the ethidium fluorescence was viewed immediately at Ex535/Em610 with the confocal microscope.

\section{Immunohistochemistry and immunofluorescence assays}

CD31 and heme oxygenase 1 (HO-1) in cardiac tissues were determined using immunofluorescence staining as previously described [55]. Briefly, sections were blocked with $5 \%$ bovine serum albumin, then incubated with primary antibody (Cell Signaling Technology, Danvers, MA, USA) overnight at $4{ }^{\circ} \mathrm{C}$. Sections were then incubated with fluorescent secondary antibody (Cell Signaling Technology, Danvers, MA, USA) at room temperature for $1 \mathrm{~h}$. Finally, the slides were counter-stained with DAPI for $30 \mathrm{~min}$, and images were acquired using a Nikon A1 confocal microscope (Nikon Corporation, Tokyo, Japan).

\section{TUNEL histochemistry}

For in situ detection of apoptosis, TdT-mediated fluorescein nucleotides (Cyanine 3-dUTP) nick-end labeling (TUNEL) was used to characterize the degree of apoptosis in cardiac tissues (Beyotime Biotechnology, Shanghai, China). Incorporated fluorescein-labeled dUTP was analyzed using a confocal microscope.

\section{Transmission electron microscope (TEM)}

Cardiac tissues were fixed in $2.5 \%$ glutaraldehyde for $48 \mathrm{~h}$ at room temperature. Then, the tissues were dehydrated and then embedded in araldite. Tissue slices $(50 \mathrm{~nm})$ were stained with lead citrate acid and uranium acetate to observe mitochondria and cardiac microvasculature by a $\mathrm{H} 600$ electron microscope (Hitachi, Tokyo, Japan) [56]. The vascular basement membrane thickness of each sample was determined by the average value of six measurements at various position. 


\section{Colonic microbiota high throughput sequencing}

Colonic contents were collected and stored at $-80^{\circ} \mathrm{C}$ until being analyzed. Total bacterial DNA was isolated from the colonic contents by the QIAamp DNA Stool Mini Kit (Qiagen, Hilden, Germany). The colonic bacterial $16 \mathrm{~S}$ rDNA gene was amplified by PCR utilizing the $341 \mathrm{~F}$ and $806 \mathrm{R}$ primers. The PCR products were purified by the QIAquick Gel Extraction Kit (Qiagen, Hilden, Germany) and sequenced on the Illumina Miseq PE250 platform (Illumina, San Diego, CA, USA) by Realbio (Realbio Technology Co, Ltd., Shanghai, China). In addition, $\beta$-diversity were analyzed by the QIIME software. Linear discriminant analysis (LDA) effect size (LEfSe) was used to characterize the marked difference in the relative abundance of microbial taxa between various groups. A heatmap was analyzed by R Cytoscape, which indicated the relativity between colonic gut microbiota and physiological index.

\section{Statistical analysis}

Data are presented as the mean \pm standard error of the mean (SEM). Differences between groups were analyzed by SPSS software (v.20.0; IBM, Armonk, NY, USA) via one-way ANOVA with Student's t test. And, Tukey's honestly significant difference test was used for the analysis of multiple comparisons.

Differences were considered to be statistically significant between various groups when $p<0.05$. The analyses were performed using GraphPad Prism software (v.7.0; GraphPad Software Inc., San Diego, CA, USA). In addition, microbial community a and $\beta$-diversity were calculated using Chao1, Shannon, and Simpson indices and Bray-Curtis distances visualized by principle coordinates analysis (PCoA). The linear discriminate analysis effect size (LEfSe) with an LDA score $>2$ and $p<0.05$.

\section{Results}

\section{Effects of Cana on hyperglycemia and IR in T2DM mice}

T2DM is related to impaired glucose tolerance and IR[57]. Chronic hyperglycemia and IR are known to play a significant role in the development of CVD among patients with T2DM [58]. SGLT2i are newly developed antidiabetic agents via inhibiting glucose reabsorption in the kidney [59]. Thus, we investigated the effect of Cana on blood glucose control, insulin sensitivity, and pancreatic protection in T2DM mice. Fasting blood glucose levels of the HFD-fed groups were markedly increased compared with that of the Control group (Fig. 1A, showed in blue color) at the 24th week, which indicated that T2DM was successfully induced by HFD-feeding. As shown in Fig. 1A and B, Cana significantly decreased fasting hyperglycemia and $\mathrm{HbA} 1 \mathrm{c}$ level compared with the Model group $(p<0.05)$, suggesting that Cana has a long-term effect on alleviating hyperglycemia. OGTT and IPITT assays at the end of the trial demonstrated that Cana treatment efficiently increase glucose tolerance (Fig. 1C and D) and improve insulin sensitivity (Fig. 1E\&F) in mice with T2DM $(p<0.05)$. The suppressed hyperinsulinemia and the lowered HOMA-IR index in Cana group compared with the Model group (Fig. $1 \mathrm{G} \& \mathrm{H})(p<0.05)$ indicate that Cana can effectively improve insulin homeostasis. 
In addition, it has been demonstrated that as diabetes developed the proliferation of pancreatic a cells increased, which resulted in an elevated plasma glucagon level and hyperglycemia [60]. Furthermore, we found that the amount of $\beta$ cells was obviously decreased, while a cells were more or less evenly distributed in the islets of T2DM mice compared with the Control group by immunofluorescence staining of insulin and glucagon (Fig. 1I). Treatment with Metf or Cana not only increased $\beta$ cells percentage and $\beta$ cells mass (Fig. 1I), it also drove a cells to the periphery of islets (Fig. 1I), which show excellent islet protection in T2DM mice. All of the above analyses confirm the anti-diabetic effects of Cana as demonstrated by regulation of blood glucose, insulin homeostasis, insulin sensitivity, and relieving pancreatic disorder in mice with T2DM.

\section{Effects of Cana on energy metabolism in T2DM mice}

After 30-weeks feeding trial, significantly different degrees of obesity were observed among various groups. The waistlines of the Model group and the Cana group were larger than the Control group but were significantly reduced after Metf treatment for six weeks (Fig. 2A), which indicate that Metf treatment can conspicuously reduce obesity degree of T2DM mice by qualitative observation while Cana treatment can't. In addition, the T2DM mice took in much more energy and gained more weight compared with the normal mice $(p<0.05)$ (Fig. 2B-D). However, Metf administration markedly prevented HFD-induced increase of body weight, body weight gain, and body mass index (BMI) (Fig. 1B-E) $(p<0.05)$, while Cana treatment couldn't. It is noteworthy that the Cana group took in much more energy compared with both the Control and the Model groups $(p<0.05)$ (Fig. 1B) in the state of taking food freely, while the energy efficiency of the Cana group was much lower than that of the Model group $(p<0.05)$ (Fig. 1F). It has been confirmed that Cana delay the rate of intestinal glucose absorption via intestinal SGLT1 inhibition and increase the urinary glucose excretion via renal SGLT2 inhibition [61], thus reducing the available energy intake. And, clinical research demonstrated that Cana can induce body weight reduction in patients with T2DM in the state of taking food with limitation [62].

\section{Effect of Cana on hematological parameters in T2DM mice}

Studies demonstrate the strong association between hematological parameters and risk of CDV, during to systemic inflammation and hypoxemia implicated in the pathophysiology mechanisms of CVD [63]. Our results (Table 1) showed that white blood cell count (WBC), platelets, and mean platelet volume (MPV) levels were conspicuously increased in T2DM mice compared with the normal mice, while Cana or Metf treatment effectively reversed this adverse situation. There is an significant association of increased MPV and platelet counts with diabetes related to endothelial dysfunction, coronary artery disease, and its vascular complications $[64,65]$. Moreover, elevated WBC is a classical inflammatory marker and associated with several CVD risk factors [66]. The above results indicate that Cana can conduct anti-CVD effect via regulating the disorderly hematological parameters in T2DM mice.

\section{Table 1 Effect of the Cana treatment on hematological parameters}




\begin{tabular}{|c|c|c|c|c|}
\hline Variable & Control & Model & Metf & Cana \\
\hline WBC $\left(\times 10^{9} / \mathrm{L}\right)$ & $4.6 \pm 0.5$ & $14.1 \pm 0.2^{*}$ & $8.1 \pm 0.9^{* \#}$ & $10 \pm 1.1^{\star \#}$ \\
\hline Monocytes $\left(\times 10^{9} / \mathrm{L}\right)$ & $0.2 \pm 0.03$ & $0.3 \pm 0.02^{*}$ & $0.6 \pm 0.04^{* \#}$ & $0.4 \pm 0.02^{* \# \&}$ \\
\hline Monocytes (\%) & $5 \pm 0.3$ & $3.8 \pm 0.25^{\star}$ & $7.4 \pm 0.5^{\star \#}$ & $2.5 \pm 0.3^{\star \# \&}$ \\
\hline Lymphocytes (x109/L) & $3.2 \pm 0.24$ & $11.9 \pm 0.9^{*}$ & $4.9 \pm 0.35^{\star \#}$ & $11.2 \pm 1.3^{\star \&}$ \\
\hline Lymphocytes (\%) & $67.4 \pm 5.6$ & $76.6 \pm 4.3$ & $60.8 \pm 5.7^{\#}$ & $83.7 \pm 7.6^{\star \# \&}$ \\
\hline Granulocytes (x109/L) & $1.3 \pm 0.09$ & $2.3 \pm 0.14^{*}$ & $2.6 \pm 0.15^{\star}$ & $3.5 \pm 0.41^{\star \# \&}$ \\
\hline Granulocytes (\%) & $27.5 \pm 2.7$ & $19.5 \pm 1.5^{\star}$ & $31.8 \pm 2.9^{\#}$ & $13.7 \pm 1.1^{\text {*\#\& }}$ \\
\hline Platelets (x109/L) & $1940.5 \pm 124.2$ & $3307 \pm 32.3^{*}$ & $2467 \pm 250.4^{\star \#}$ & $2707 \pm 265.1^{* \#}$ \\
\hline PDW & $17.4 \pm 1.2$ & $17.4 \pm 1.3$ & $18.0 \pm 1.5$ & $19.3 \pm 1.2$ \\
\hline $\mathrm{RBC}\left(\mathrm{x} 10^{12} / \mathrm{L}\right)$ & $4.1 \pm 0.4$ & $5.1 \pm 0.3^{*}$ & $4.49 \pm 0.39$ & $1.4 \pm 0.11^{\star \# \&}$ \\
\hline HCT (\%) & $29.3 \pm 2.1$ & $24.5 \pm 1.7$ & $21.1 \pm 1.3^{*}$ & $7.3 \pm 0.5^{\star \# \&}$ \\
\hline $\operatorname{HGB}(g / L)$ & $115.3 \pm 10.5$ & $138.7 \pm 14.2^{*}$ & $123.7 \pm 13.2$ & $139.7 \pm 14.1^{*}$ \\
\hline $\operatorname{MCV}(f L)$ & $49.5 \pm 5$ & $48.5 \pm 5.0$ & $48.7 \pm 3.6$ & $52.7 \pm 4.8$ \\
\hline RDW (\%) & $37.8 \pm 3.2$ & $38.5 \pm 2.8$ & $38.4 \pm 3.4$ & $37.0 \pm 2.8$ \\
\hline MPV (fL) & $4.75 \pm 0.5$ & $7.56 \pm 0.8^{*}$ & $6 \pm 0.7^{\star \#}$ & $6.3 \pm 0.7^{\star \#}$ \\
\hline
\end{tabular}

PDW: platelet distribution width; RBC: red blood cell count; HCT: hematocrit; HGB: hemoglobin; MCV: mean corpuscular volume; RDW: red cell distribution width. Data are expressed as mean \pm SEM. * $p<$ 0.05 , compared with the Control group; \# $p<0.05$, compared with the Model group; $\& p<0.05$, the Cana group compared with the Metf group.

\section{Effects of Cana on hyperlipemia in T2DM mice}

Evidences demonstrate that increased concentration of cholesterol and TG is an causal risk factor for CVD [67]. And, hyperlipidemia is characterized by increased lipid accumulation in serum, which is a strong risk factor for CVD and T2DM related coronary artery disease $[68,69]$. Thus, managing hyperlipidemia is an effective way to prevent CVD. Our results showed that HFD feeding significantly increased the levels of TC, TG, and LDL-C in the serum compared with the NCD-fed mice $(p<0.05)$ (Fig. $3 A-C)$. After treatment for six weeks, the serum TG, TC, and LDL-C levels in Cana group were markedly reduced compared with the Model group $(p<0.05)$, and the therapeutic effect was comparable to the 
Metf group (Fig. 3A-C). In addition, serum HDL-C level increased in the Cana and the Metf groups compared with the Model group (Fig. 3D) $(p<0.05)$. Atherogenic index of plasma (AIP) and arteriosclerosis index (AI) values increase with the elevated CVD risk, which are also highly sensitive markers of lipoprotein profiles in CVD patients [70]. And, Cana decrease the AIP and AI levels in T2DM mice (Fig. 3E\&F), which indicate that Cana can effectively alleviate CVD induced by hyperlipidemia in mice with T2DM. In addition, Oil red $\mathrm{O}$ staining indicated that abnormal lipid accumulation was observed in the Model group as compared to the Control group, while treated with Cana alleviated the lipid accumulation in cardiac tissues (Fig. 3G). Taken together, the above results demonstrate that Cana can perform anti-CVD effect via suppressing lipid accumulation in serum and cardiac tissue in T2DM mice.

\section{Effects of Cana on oxidative stress and systematic inflammation in T2DM mice}

Oxidative stress was increased in T2DM and this appeared to underlie the development of T2DM and diabetic complications such as CVD [71]. Oxidative stress is known as a major contributor to endothelial dysfunction [72], which is a key precursor to the development of CVD [73]. As shown in Fig. 4A, the area and intensity of fluorescence from DHE oxidation were notably increased in the Model group compared to that of the Control group $(p<0.05)$, while Cana or metformin treatments markedly reversed this situation $(p<0.05)$. Notable decreases in the activities of SOD and GSH, while increasing MDA content in the heart of T2DM mice compared with the normal mice (Fig. 4B-D). However, the above situation was conspicuously reversed by Cana supplementation, implying that Cana can effectively relieve myocardial oxidative stress (Fig. 4B-D). Importantly, TEM evaluation revealed marked cardiomyocyte dissolution, muscular fiber twisting, intercalated disc blurred, $Z$ line disappearance, and some myofilaments were flocky in appearance and showed no cross striations in the myocardial tissue of diabetic mice. In addition, mitochondrial crista deformation and damage, mitochondrial autophagy (red arrows presented in Fig. 4E, vacuoles with black residue), and fat drop (yellow arrows presented in Fig. 4E, vacuoles without black spots) were significantly presented in the Model group compared with the Control group. And, the effects of which were greatly attenuated by Cana or Metf treatment (Fig. 4E), which are beneficial for the suppression of mitochondrial ROS production and subsequently oxidative stress [74].

Chronic inflammation is also an established mediator of vascular dysfunction in individuals with T2DM [75-77]. Through the inflammatory processes, the initial lesion of atherosclerosis and CVD is formed [78]. We found that HFD feeding elevated systematic inflammation state as demonstrated by increased serum levels of inflammatory cytokines including TNFa, MCP-1, and IL- 6 compared with the Control group, whereas Cana administration markedly suppressed this increase of pro-inflammatory cytokines (Fig. 4F-H). Besides, $\mathrm{HO}-1$ level was markedly decreased in the Model group compared with the Control group $(p<0.05)$, while Cana or Metf treatment effective elevated the HO-1 level in T2DM mice ( $p<$ 0.05). Upregulation of HO-1 expression plays an important role in the protective response against oxidative injury and inflammatory effects [79], which proposes that HO-1 is a promising target protein for therapeutic intervention in CVD. In general, the above results indicate that Cana can effectively alleviate 
oxidative stress and systematic inflammation by protecting myocardial structural and mitochondrial homeostasis.

\section{Effects of Cana on cardiovascular abnormalities in T2DM mice}

Normal vasculature is crucial to cardiac function and CVD, which is susceptible to hyperglycemia [80]. CD31 plays an important role in endothelial protection, which alleviates the apoptosis of vascular endothelial cells [81]. HFD induced T2DM triggered an overtly drop in the number of CD31 positive microvessels compared with the normal mice (Fig. 5A), while the unfavorable state was reversed by Cana or Metf treatment so as to play a good role in protecting the vascular endothelium. In addition, diabetesinduced changes in microvascular morphology including fibrosis (Fig. 5B) and basement membrane thickening (Fig. 5C), which were largely ameliorated by Cana administration. These data demonstrated a cardioprotective property of Cana by maintaining cardiovascular structural homeostasis in T2DM mice.

\section{Effects of Cana on myocardial injury in T2DM mice}

Damage to microvessel integrity and cardiac microvascular endothelial cell are considered the initial step in vascular complications in diabetes [82]. Diabetes-induced effects (such as turbulent blood flow and capillary blockage) raised the accumulation of erythrocytes in the microvessel [74], while Cana considerably retarded this negative situation (red arrows presented in Fig. 6A). Additionally, Metf or Cana treatment inhibited the diabetes-induced TUNEL positive cells increasing (red arrows presented in Fig. $6 \mathrm{~B})$, supporting the pro-survival capacity of Cana on hyperglycemia-mediated cardiac microvascular endothelial cell apoptosis. These data revealed the beneficial effects of Cana on microvessel integrity in diabetes, favoring a significant decrease for the risk of cardiac microvascular endothelial cell dysfunction or death in diabetic hearts.

Cardiac troponin is used to diagnose myocardial infarction, and the increased concentration of cardiac troponin indicates the elevated risk for adverse outcome in individual [83]. In addition, cTn I, an important subunits of cardiac troponin, is recognized as marker of myocardial damage [84]. Studies also demonstrated that high level of SCD40L indicates enhanced inflammatory responses, heightened risk of death and myocardial infarction in patients [85-87]. In our study, levels of serous CTn I and SCD40L in T2DM mice were significantly increased compared with that in the Control group (Fig. 6 C\&D), while Cana treatment markedly improved the situation. The above results demonstrate the salient effects of Cana on alleviating myocardial injury in T2DM mice.

\section{Cana modulated gut microbiota at different taxonomic levels}


High-throughput 16S rDNA sequencing was applied to elucidate the effect of Cana on colonic microbiota in HFD-induced T2DM. As shown in Table S1, a-diversity (e.g. Good's coverage, Chao1, Shannon and Simpson indices) of the colonic flora in Cana-treated group was not significantly different compared with that in the Control and the Model groups. In addition, evaluation of $\beta$-diversity based on nMDS ordination plot (Fig. S2A), weighted UniFrac heatmap (Fig. S2B), and weighted PCoA analysis (Fig. 7A) showed that there were significant differences in colonic flora between the Cana treatment group and the Model group, and the microbial community of the Cana group was similarly to that of the Control group. At the phylum level (Fig. 7B), the Cana group had a higher abundance of Bacteroidetes with a lower abundance of Firmicutes, and an increased ratio of Firmicutes/Bacteroidetes (Fig. 7C) compared with that of the Model group. A significant increase in the abundance of Proteobacteria was induced in the Model group and was significantly reduced in the Cana group, however, not reversed by Metf treatment (Fig. 7D). In addition, administration of Cana remarkably enriched the abundance of Actinobacteria and decreased the abundance of Deferribacteres in mice with T2DM (Fig. 7E\&F). The abundance analysis at genus level showed that there were significant differences in flora types and abundance in T2DM mice after Cana treatment (Fig. 7G). Furthermore, Cana regulated the disorder of microbial community in genus level as follows: 1. increasing the relative abundance of Olsenella, Alistipes, and Alloprevotella (Fig. 7l, $\mathrm{J} \& \mathrm{~K}$ ), which may be associated with a healthier microbiome, as suggested previously [88]; 2. decreasing the relative abundance of Helicobacter and Mucispirillum (Fig. 7H\&L), which was positively correlated with TNFa and LPS contents [89]. Altogether, the above results indicated that intestinal ecosystem in mice with diabetic CVD were adjusted to a relatively normal state by oral administration of Cana.

\section{Key phylotypes of gut microbiota in response to Cana treatment}

To further elucidate the effects of Cana treatment on the regulation of various bacterial taxa in colon, a pairwise comparison between the Model and the Cana groups was conducted using LEfSe analysis. It revealed that Cana decreased the level of Firmicutes, including Sporacetigenium, Veillonella, and Clostridium Xl, while markedly increasing Bacteroidetes, including short-chain fatty acid producers such as Alloprevotella and Bacteroides, compared with the Model group $(p<0.05)$ (Fig. 8A\&B). And, the Canatreated group was characterized by a higher amount of Porphyromonadaceae, Rikenellaceae, and Corynebacteriaceae, while a decreased in Desulfovibrionaceae and Veillonellaceae. At the genus level, the Cana group was marked by a dramatic increase in Alistipes, Roseburia, and Corynebacterium (Fig. 8B), which can increse the production of short-chain fatty acids (SCFAs) [90, 91]. Meanwhile, Paraprevotella, Veillonella, Sporacetigenium, and Intestinimonas were significantly reduced after Cana treatment (Fig. 8B). In general, the taxonomical distribution within groups at phylum, family, and genus levels for the colonic samples revealed the divergent composition of communities after Cana treatment in mice with diabetic CVD.

Using the Spearman's correlation analysis, relationships of physiological index and bacterial abundance were clarified (Fig. 8C-E). At the genus level, Parabacteroides and Alistipes were positively 
correlated with CAT and SOD, while negatively correlated with MDA, ROS, IL-1 $\beta, T N F a$, LBP, and MCP-1 contents, and their relative abundances were raised after Cana treatment compared with the Model group. In addition, Clostridium XI, Veillonella, and Paraprevotella were negatively correlated with CAT and SOD, while positively related to MDA, ROS, and the above inflammatory cytokines levels. Meanwhile, Alistipes and Roseburia were negatively correlated with LDL-C, TC and TG contents, while positively related to HDL$C$ level. And, Cana treatment caused an increase in the relative abundances of Alistipes and Roseburia in mice with diabetic CVD. Moreover, Paraprevotella, Veillonella, Clostridium XI, Barnesiella presented positively correlated with LDL-C, TC and TG contents, while negatively related to HDL-C level. These findings suggest that alterations of the gut microbiome after Cana treatment lead to the decrease of oxidative stress level, systemic inflammation, and lipid accumulation in mice with diabetic CVD. Collectively, these data reinforce the link between gut dysbiosis and diabetic CVD, and indicate that Cana can manipulate gut microbiota and attenuate the CVD complications of T2DM.

\section{Discussion}

A clinical research indicated that Cana can lower the risk of cardiovascular events in patients with T2DM [40], and yet the underlying mechanisms was still unclear. In an environment of chronic hyperglycemia, vascular function is disrupted due to elevations in ROS and inflammation, which play a pivotal role in the development of diabetic CVD [92]. Our present study showed that Cana can markedly relieve the oxidative stress and inflammation state, which may due to the Cana maintained mitochondrial homeostasis of cardiac tissue in mice with diabetic CVD. Mitochondrial dysfunction resulted in the overproduction of ROS, and cardiovascular function can be improved by relieving mitochondrial dysfunction and its related oxidative stress and systemic inflammatory state [93]. Further understanding of oxidative stress as well as its downstream effects on cellular function will be conducive to identify more specific targets for CVD treatment [15]. Thus, mitochondria may be a potential target for diabetic CVD treatment, while further verification is required.

It has been verified that gut microbiota and its metabolites affect the autophagy and damage of mitochondria, thus regulating the mitochondrial function of host [94]. Interestingly, Cana treatment decreased the level of Firmicutes, while markedly increasing Bacteroidetes, including SCFAs producers such as Alloprevotella and Bacteroides, which were benefit for alleviating hypertension and atherosclerosis [95]. In addition, Cana administration was marked by a dramatic increase in Roseburia, Alloprevotella, and Bacteroides, which can elevate the production of SCFAs. Furthermore, Spearman's correlation analysis demonstrated that the colonic microbiota composition altered by Cana treatment was related with the blood lipid, oxidative stress, and inflammation. Therefore, Cana subtly altered microbiota composition in T2DM mice with CVD, which contributed to the improvement of mitochondrial homeostasis and the progress of CVD [96].

In summary, Cana can effectively alleviate the risk factors for diabetic CVD such as oxidative stress, inflammation, hyperlipemia, atherosclerosis, mitochondrial disorder, thickness of vascular basement membrane, and myocardial injury. And, the improvements of myocardial mitochondrial and gut 
microbiota homeostasis, may represent an important mechanism underlying the cardiovascular benefits of Cana treatment (Fig. 9). Thus, Cana is a promising agent for relieving T2DM related CVD.

\section{Conclusions And Limitations}

In summary, our results confirm that Cana can effectively ameliorate diabetic CVD induced by HFD feeding. Compared with the effects of Metf $(225 \mathrm{mg} / \mathrm{kg} / \mathrm{d})$, Cana $(50 \mathrm{mg} / \mathrm{kg} / \mathrm{d})$ executed comparative effects on alleviating diabetic CVD while played a better regulatory role in regulating gut microbiota to a direction that favored the host. We found that Cana significantly relieved the oxidative stress and inflammation in heart by maintaining myocardial mitochondrial homeostasis in mice with diabetic CVD. Cana profoundly improved lipid homeostasis in HFD-fed mice, thus decreased the risk of atherosclerosis. Additionally, Cana treatment could maintain a relatively normal haemogram, and reducing the thickness of vascular basement membrane and heart injury. Importantly, Cana also alleviated diabetic CVD by restoring colonic microbiota dysbiosis, which can conspicuously inhibit oxidative stress state, systemic inflammation, lipid accumulation, and maintain mitochondrial homeostasis. Altogether, our study presents a novel therapeutic role for Cana in CVD mainly by the protective effect of myocardial mitochondria and the role of gut microbiota modulation.

Several limitations to this current study should be noted. First, further research to clarify the precise molecular mechanism of the associated changes in colonic microbiota that lead to the improvement of diabetic CVD is still necessary. Second, the relationship between microbiota and CVD should be further clarified utilizing fecal bacteria transplantation in antibiotic treated or germ-free mice model, which is necessary to be comprehensively addressed in future research. In addition, the most important probiotic strains in alleviating diabetic CVD after Cana treatment were not clarified.

\section{Abbreviations}

T2DM: type 2 diabetes mellitus; CVD: cardiovascular disease; IR: insulin resistance; Metf: metformin; SGLT2: sodium-glucose cotransporter 2; SGLT2i: sodium glucose cotransporter 2 inhibitors; Cana: canagliflozin; OGTT: oral glucose tolerance test; IPITT: intraperitoneal insulin tolerance test; ROS: reactive oxygen species; AUC: area under the curve; WBC: white blood cell count; RBC: red blood cell count; HGB: hemoglobin; HCT: hematocrit; MCV: mean corpuscular volume; RDW: red cell distribution width; PDW: platelet distribution width; MPV, mean platelet volume; Al: atherogenic index; AIP: atherogenic index of plasma; FBG: Fasting blood glucose; HbA1c: hemoglobin A1c; TC: total cholesterol; TG: triglycerides; LDLC: low-density lipoprotein cholesterol; HDL-C: high-density lipoprotein cholesterol; SOD: superoxide dismutase; GSH: glutathione; CAT: catalase; MDA: malondialdehyde; HbA1c: hemoglobin A1c; HOMA-IR: homeostasis model assessment-insulin resistance; MCP-1: monocyte chemotactic protein 1; IL-6: interleukin-6; TNFa: tumor necrosis factor a; Tn I: cardiac troponin I; sCD40L: soluble cluster of differentiation 40 ligand; AMPK: AMP-activated protein kinase; TEM: transmission electron microscope; SEM: standard error of the mean; LefSe: linear discriminate analysis effect size; DHE: dye dihydroethidium; Dapa: dapagliflozin; short-chain fatty acids: SCFAs. 


\section{Declarations}

\section{Competing interests}

The authors declare that they have no competing interests.

\section{Consent for publication}

All authors gave their consent for publication.

\section{Availability of data and materials}

Please contact author for data requests.

\section{Ethics approval and consent to participate}

All of our investigation protocols were approved by the Experimental Animal Care and Use Committee of Ocean University of China and complied with the Guide for the Care and Use of Laboratory Animals (NIH publication, 8th edition, 2011).

\section{Funding}

This work was supported by National Natural Science Foundation of China (81991522, 21807094), National Science and Technology Major Project of China (2018ZX09735-004), Shandong Provincial Major Science and Technology Innovation Project (2018SDKJ0404), Taishan Scholar Climbing Project (TSPD20210304), Fundamental Research Funds for the Central Universities (202042005), and Natural Science Foundation of Shandong Province (ZR2017BC007).

\section{Authors' Contributions}

Xueliang Wang conducted the research and wrote the paper. Di Liu and Zhe Wang helped with the animal experiment and biochemical analysis. Xueliang Wang, Hao Jiang, and Guangli Yu analyzed experimental data. Xueliang Wang, Hao Jiang, Chao Cai, Guoyun Li, and Guangli Yu contributed to the manuscript revision. Guangli Yu and Hao Jiang designed and directed the project. All authors read and approved the final manuscript.

\section{Acknowledgments}


Not applicable.

\section{References}

1. Chen $\mathrm{CC}$, Susanto $\mathrm{H}$, Chuang WH, Liu TY, Wang $\mathrm{CH}$. Higher serum betatrophin level in type 2 diabetes subjects is associated with urinary albumin excretion and renal function. Cardiovasc Diabetol. 2016;15(1):3.

2. DeFronzo RA, Ferrannini E, Groop L, Henry RR, Herman WH, Holst JJ, et al. Type 2 diabetes mellitus. Nat Rev Dis Primers. 2015;1:15019.

3. Figueiredo VN, Yugar-Toledo JC, Martins LC, Martins LB, de Faria APC, de Haro Moraes C, et al. Vascular stiffness and endothelial dysfunction: correlations at different levels of blood pressure. Blood pressure. 2012;21(1):31-38.

4. Stevens SL, Wood S, Koshiaris C, Law K, Glasziou P, Stevens RJ, et al. Blood pressure variability and cardiovascular disease: systematic review and meta-analysis. BMJ. 2016;354:i4098.

5. Brindle P, Beswick A, Fahey T, Ebrahim S. Accuracy and impact of risk assessment in the primary prevention of cardiovascular disease: a systematic review. Heart. 2006;92(12):1752-1759.

6. Haffner SM, Lehto S, Rönnemaa T, Pyörälä K, Laakso M. Mortality from coronary heart disease in subjects with type 2 diabetes and in nondiabetic subjects with and without prior myocardial infarction. New Engl J Med. 1998;339(4):229-234.

7. Fox CS, Golden SH, Anderson C, Bray GA, Burke LE, De Boer IH et al. Update on prevention of cardiovascular disease in adults with type 2 diabetes mellitus in light of recent evidence: a scientific statement from the American Heart Association and the American Diabetes Association. Circulation. 2015;132(8):691-718.

8. Gerstein H, Pogue J, Mann J, Lonn E, Dagenais G, McQueen M, et al. The relationship between dysglycaemia and cardiovascular and renal risk in diabetic and non-diabetic participants in the HOPE study: a prospective epidemiological analysis. Diabetologia. 2005;48(9):1749-1755.

9. Skyler JS, Bergenstal R, Bonow RO, Buse J, Deedwania P, Gale EA, et al. Intensive glycemic control and the prevention of cardiovascular events: implications of the ACCORD, ADVANCE, and VA diabetes trials: a position statement of the American Diabetes Association and a scientific statement of the American College of Cardiology Foundation and the American Heart Association. J Am Coll Cardiol. 2009;53(3):298-304.

10. Group AC. Intensive blood glucose control and vascular outcomes in patients with type 2 diabetes. New Engl J Med. 2008;358(24):2560-2572.

11. Tietge UJ. Hyperlipidemia and cardiovascular disease: inflammation, dyslipidemia, and atherosclerosis. Curr Opin Lipidol. 2014;25(1):94-95.

12. Varbo A, Benn M, Tybjærg-Hansen A, Nordestgaard BG. Elevated remnant cholesterol causes both low-grade inflammation and ischemic heart disease, whereas elevated low-density lipoprotein 
cholesterol causes ischemic heart disease without inflammation. Circulation. 2013;128(12):12981309.

13. Braunwald E. Biomarkers in heart failure. New Engl J Med. 2008;358(20):2148-2159.

14. Nishikawa T, Araki E. Impact of mitochondrial ROS production in the pathogenesis of diabetes mellitus and its complications. Antioxid Redox Sign. 2007;9(3):343-353.

15. Kayama Y, Raaz U, Jagger A, Adam M, Schellinger IN, Sakamoto M, et al. Diabetic cardiovascular disease induced by oxidative stress. Int J Mol Sci. 2015;16(10):25234-25263.

16. Jain KS, Kathiravan M, Somani RS, Shishoo CJ. The biology and chemistry of hyperlipidemia. Bioorgan Med Chem. 2007;15(14):4674-4699.

17. Aronson D, Rayfield EJ. How hyperglycemia promotes atherosclerosis: molecular mechanisms. Cardiovasc Diabetol. 2002;1(1):1.

18. da Silva A, Caldas APS, Hermsdorff HHM, Bersch-Ferreira ÂC, Torreglosa CR, Weber B, et al. Triglyceride-glucose index is associated with symptomatic coronary artery disease in patients in secondary care. Cardiovasc Diabetol. 2019;18(1):89.

19. Rosenwasser RF, Sultan S, Sutton D, Choksi R, Epstein BJ. SGLT-2 inhibitors and their potential in the treatment of diabetes. Diabet Metab Synd OB. 2013;6:453.

20. Yang XP, Lai D, Zhong XY, Shen HP, Huang YL. Efficacy and safety of canagliflozin in subjects with type 2 diabetes: systematic review and meta-analysis. Eur J Clin Pharmacol. 2014;70(10):1149-1158.

21. Sun YN, Zhou Y, Chen X, Che WS, Leung SW. The efficacy of dapagliflozin combined with hypoglycaemic drugs in treating type 2 diabetes mellitus: meta-analysis of randomised controlled trials. BMJ open. 2014;4(4):e004619.

22. Liakos A, Karagiannis T, Athanasiadou E, Sarigianni M, Mainou M, Papatheodorou K, et al. Efficacy and safety of empagliflozin for type 2 diabetes: a systematic review and meta-analysis. Diabetes Obes Metab. 2014;16(10):984-993.

23. Xiang B, Zhao X, Zhou X. Cardiovascular benefits of sodium-glucose cotransporter 2 inhibitors in diabetic and nondiabetic patients. Cardiovasc Diabetol. 2021;20(1):78.

24. Dziuba J, Alperin P, Racketa J, lloeje U, Goswami D, Hardy E, et al. Modeling effects of SGLT-2 inhibitor dapagliflozin treatment versus standard diabetes therapy on cardiovascular and microvascular outcomes. Diabetes Obes Metab 2014;16(7):628-635.

25. Lee DM, Battson ML, Jarrell DK, Hou S, Ecton KE, Weir TL, et al. SGLT2 inhibition via dapagliflozin improves generalized vascular dysfunction and alters the gut microbiota in type 2 diabetic mice. Cardiovasc Diabetol. 2018;17(1):62.

26. Cefalu WT. Paradoxical insights into whole body metabolic adaptations following SGLT2 inhibition. J Clin Invest. 2014;124(2):485-487.

27. Dixon JB. Obesity in 2015: advances in managing obesity. Nat Rev Endocrinol. 2016;12(2):65.

28. Zinman B, Wanner C, Lachin J, Fitchett D, Bluhmki E, Hantel S, et al. Empagliflozin, Cardiovascular Outcomes, and Mortality in Type 2 Diabetes. New Engl J Med. 2015;373(22):2117. 
29. Forslund K, Hildebrand F, Nielsen T, Falony G, Le Chatelier E, Sunagawa S, et al. Disentangling type 2 diabetes and metformin treatment signatures in the human gut microbiota. Nature. 2015;528(7581):262.

30. Boulangé CL, Neves AL, Chilloux J, Nicholson JK, Dumas ME. Impact of the gut microbiota on inflammation, obesity, and metabolic disease. Genome Med. 2016;8(1):1-12.

31. Battson ML, Lee DM, Jarrell DK, Hou S, Ecton KE, Weir TL, et al. Suppression of gut dysbiosis reverses Western diet-induced vascular dysfunction. Am J Physiol-Endoc M. 2017;314(5):E468-E477.

32. Vikram A, Kim YR, Kumar S, Li Q, Kassan M, Jacobs JS, et al. Vascular microRNA-204 is remotely governed by the microbiome and impairs endothelium-dependent vasorelaxation by downregulating Sirtuin1. Nat Commun. 2016;7:12565.

33. Montandon S, Jornayvaz F. Effects of antidiabetic drugs on gut microbiota composition. Genes. 2017;8(10):250.

34. Caparrós-Martín JA, Lareu RR, Ramsay JP, Peplies J, Reen FJ, Headlam HA, et al. Statin therapy causes gut dysbiosis in mice through a PXR-dependent mechanism. Microbiome. 2017;5(1):95.

35. Maier L, Pruteanu M, Kuhn M, Zeller G, Telzerow A, Anderson EE, et al. Extensive impact of nonantibiotic drugs on human gut bacteria. Nature. 2018;555(7698):623.

36. Steven S, Oelze M, Hanf A, Kröller-Schön S, Kashani F, Roohani S, et al. The SGLT2 inhibitor empagliflozin improves the primary diabetic complications in ZDF rats. Redox Biol. 2017;13:370-385.

37. Zinman B, Lachin JM, Inzucchi SE. Empagliflozin, cardiovascular outcomes, and mortality in type 2 diabetes. New Engl J Med. 2016;374(11):1094.

38. Hawley SA, Ford RJ, Smith BK, Gowans GJ, Mancini SJ, Pitt RD, et al. The Na ${ }^{+} /$glucose co-transporter inhibitor canagliflozin activates AMP-activated protein kinase by inhibiting mitochondrial function and increasing cellular AMP levels. Diabetes. 2016;65(9):2784.

39. Garvey WT, Van Gaal L, Leiter LA, Vijapurkar U, List J, Cuddihy R, et al. Effects of canagliflozin versus glimepiride on adipokines and inflammatory biomarkers in type 2 diabetes. Metabolism. 2018;85:3237.

40. Perkovic V, Jardine MJ, Neal B, Bompoint S, Heerspink HJL, Charytan DM, et al. Canagliflozin and Renal Outcomes in Type 2 Diabetes and Nephropathy. New Engl J Med. 2019;380:2295-2306.

41. Guthrie R. Canagliflozin and Cardiovascular and Renal Events in Type 2 Diabetes. New Engl J Med. 2018;130(2):149-153.

42. Mahaffey KW, Neal B, Perkovic V, De ZD, Fulcher G, Erondu N, et al. Canagliflozin for Primary and Secondary Prevention of Cardiovascular Events: Results From the CANVAS Program (Canagliflozin Cardiovascular Assessment Study). Circulation. 2018;137(4):323-334.

43. Mishima E, Fukuda S, Kanemitsu Y, Saigusa D, Mukawa C, Asaji K, et al. Canagliflozin reduces plasma uremic toxins and alters the intestinal microbiota composition in a chronic kidney disease mouse model. Am J Physiol-Renal. 2018;315(4):824-833. 
44. Fung CSC, Wan EYF, Wong CKH, Jiao FF, Chan AKC. Effect of metformin monotherapy on cardiovascular diseases and mortality: a retrospective cohort study on Chinese type 2 diabetes mellitus patients. Cardiovasc Diabetol. 2015;14(1):137.

45. Jaikumkao K, Pongchaidecha A, Chueakula N, Thongnak L, Wanchai K, Chatsudthipong V, et al. Renal outcomes with sodium glucose cotransporter 2 (SGLT2) inhibitor, dapagliflozin, in obese insulin-resistant model. BBA. 2018;1864(6):2021-2033.

46. Tholngnak L, Pongchaidecha A, Jaikumkao K, Chatsudthipong V, Chattipakorn N, Lungkaphin A. The additive effects of atorvastatin and insulin on renal function and renal organic anion transporter 3 function in diabetic rats. Sci Rep. 2017;7(1):13532.

47. Arjinajarn P, Pongchaidecha A, Chueakula N, Jaikumkao K, Chatsudthipong V, Mahatheeranont S, et al. Riceberry bran extract prevents renal dysfunction and impaired renal organic anion transporter 3 (Oat3) function by modulating the PKC/Nrf2 pathway in gentamicin-induced nephrotoxicity in rats. Phytomedicine. 2016;23(14):1753-1763.

48. Strissel KJ, Stancheva Z, Miyoshi H, Perfield JW, DeFuria J, Jick Z, et al. Adipocyte death, adipose tissue remodeling, and obesity complications. Diabetes. 2007;56(12):2910-2918.

49. Goldner J. A modification of the Masson trichrome technique for routine laboratory purposes. Am J Pathol. 1938;14(2):237.

50. Fischer AH, Jacobson KA, Rose J, Zeller R. Hematoxylin and eosin staining of tissue and cell sections. Cold Spring Harbor Protocols. 2008; 2008(5):pdb. prot4986.

51. Wang L, Fouts DE, Stärkel P, Hartmann P, Chen P, Llorente $C$, et al. Intestinal REG3 lectins protect against alcoholic steatohepatitis by reducing mucosa-associated microbiota and preventing bacterial translocation. Cell Host Microbe. 2016;19(2):227-239.

52. Fei $\mathrm{N}$, Zhao L. An opportunistic pathogen isolated from the gut of an obese human causes obesity in germfree mice. ISME J. 2013;7(4):880.

53. Fraulob JC, Ogg-Diamantino R, Fernandes-Santos C, Aguila MB, Mandarim-de-Lacerda CA. A mouse model of metabolic syndrome: insulin resistance, fatty liver and non-alcoholic fatty pancreas disease (NAFPD) in C57BL/6 mice fed a high fat diet. J Clin Biochem Nutr. 2010;46(3):212.

54. Zheng Y, Liu T, Wang Z, Xu Y, Zhang Q, Luo D. Low molecular weight fucoidan attenuates liver injury via SIRT1/AMPK/PGC1a axis in db/db mice. Int J Biol Macromol. 2018;112:929-936.

55. El-Azab MF, Hazem RM, Moustafa YM. Role of simvastatin and/or antioxidant vitamins in therapeutic angiogenesis in experimental diabetic hindlimb ischemia: effects on capillary density, angiogenesis markers, and oxidative stress. Eur J Pharmacol. 2012;690(1-3):31-41.

56. Das N, Mandala A, Naaz S, Giri S, Jain M, Bandyopadhyay D, et al. Melatonin protects against lipidinduced mitochondrial dysfunction in hepatocytes and inhibits stellate cell activation during hepatic fibrosis in mice. J Pineal Res. 2017;62(4):e12404.

57. Prior JO, Quiñones MJ, Hernandez-Pampaloni M, Facta AD, Schindler TH, Sayre JW, et al. Coronary circulatory dysfunction in insulin resistance, impaired glucose tolerance, and type 2 diabetes mellitus. Circulation. 2005;111(18):2291-2298. 
58. Zhang Y, Hu G, Yuan Z, Chen L. Glycosylated hemoglobin in relationship to cardiovascular outcomes and death in patients with type 2 diabetes: a systematic review and meta-analysis. PloS One. 2012;7(8):e42551.

59. Vallon V, Platt KA, Cunard R, Schroth J, Whaley J, Thomson SC, et al. SGLT2 mediates glucose reabsorption in the early proximal tubule. J Am Soc Nephrol. 2011;22(1):104-112.

60. Liu Z, Kim W, Chen Z, Shin YK, Carlson OD, Fiori JL, et al. Insulin and glucagon regulate pancreatic acell proliferation. PloS One 2011, 6(1):e16096.

61. Polidori D, Sha S, Mudaliar S, Ciaraldi TP, Ghosh A, Vaccaro N, et al. Canagliflozin lowers postprandial glucose and insulin by delaying intestinal glucose absorption in addition to increasing urinary glucose excretion: results of a randomized, placebo-controlled study. Diabetes Care. 2013;36(8):2154-2161.

62. Cefalu WT, Stenlöf K, Leiter LA, Wilding JP, Blonde L, Polidori D, et al. Effects of canagliflozin on body weight and relationship to $\mathrm{HbA} 1 \mathrm{c}$ and blood pressure changes in patients with type 2 diabetes. Diabetologia. 2015;58(6):1183-1187.

63. Fan L, Gui L, Chai EQ, Wei CJ. Routine hematological parameters are associated with short- and longterm prognosis of patients with ischemic stroke. J Clin Lab Anal. 2018;32(2):e22244.

64. Jabeen F, Fawwad A, Rizvi HA, Alvi F. Role of platelet indices, glycemic control and hs-CRP in pathogenesis of vascular complications in type-2 diabetic patients. Pak J Med Sci. 2013;29(1):152156.

65. Lippi G, Salvagno GL, Nouvenne A, Meschi T, Borghi L, Targher G. The mean platelet volume is significantly associated with higher glycated hemoglobin in a large population of unselected outpatients. Prim Care Diabetes. 2015;9(3):226-230.

66. Yayla C, Gayretli Yayla K, Acar B, Unal S, Ertem AG, Akboga MK, et al. White Blood Cell Subtypes and Ratios in Cardiovascular Disease. Angiology. 2017;68(7):651.

67. Nordestgaard BG, Varbo A. Triglycerides and cardiovascular disease. Lancet. 2014;384(9943):626635.

68. Centers for Disease Control and Prevention: National ambulatory medical care survey: 2010 summary tables. Available at http://www cdc gov/nchs/data/ahcd/namcs summary/2010 namcs web tables pdf 2010.

69. Carr MC, Brunzell JD. Abdominal obesity and dyslipidemia in the metabolic syndrome: importance of type 2 diabetes and familial combined hyperlipidemia in coronary artery disease risk. J Clin Endocr Metab. 2004;89(6):2601-2607.

70. Dobiasova M. AIP-Atherogenic index of plasma as a significant predictor of cardiovascular risk: From research to practice. Vnitrni Lekarstvi. 2006;52(1):64-71.

71. Tangvarasittichai S. Oxidative stress,insulin resistance,dyslipidemia and type 2 diabetes mellitus. World J Diabetes. 2015;6(3):456-480.

72. Magenta A, Greco S, Gaetano C, Martelli F. Oxidative stress and microRNAs in vascular diseases. Int J Mol Sci. 2013;14(9):17319-17346. 
73. Rocha M, Apostolova N, Hernandez-Mijares A, Herance R, M Victor V. Oxidative stress and endothelial dysfunction in cardiovascular disease: mitochondria-targeted therapeutics. Curr Med Chem. 2010;17(32):3827-3841.

74. Zhou H, Wang S, Zhu P, Hu S, Chen Y, Ren J. Empagliflozin rescues diabetic myocardial microvascular injury via AMPK-mediated inhibition of mitochondrial fission. Redox Biol. 2018;15:335-346.

75. Du B, Ouyang A, Eng JS, Fleenor BS. Aortic perivascular adipose-derived interleukin- 6 contributes to arterial stiffness in low-density lipoprotein receptor deficient mice. Am J Physiol-Heart C. 2015;308(11):1382-1390.

76. Zhang J, Xia L, Zhang F, Zhu D, Xin C, Wang H, et al. A novel mechanism of diabetic vascular endothelial dysfunction: Hypoadiponectinemia-induced NLRP3 inflammasome activation. BBA-Mol Basis Dis. 2017;1863(6):1556-1567.

77. de Jager J, Dekker JM, Kooy A, Kostense PJ, Nijpels G, Heine RJ,et al. Endothelial dysfunction and low-grade inflammation explain much of the excess cardiovascular mortality in individuals with type 2 diabetes: the Hoorn Study. Arterioscl Throm Vas. 2006;26(5):1086-1093.

78. Libby P. Inflammation and cardiovascular disease mechanisms. Am J Clin Nutr. 2006;83(2):456-460.

79. Lee TS, Chau LY. Heme oxygenase-1 mediates the anti-inflammatory effect of interleukin-10 in mice. Nat Med. 2002;8:240-246.

80. Pinter E, Mahooti S, Wang Y, Imhof BA, Madri JA. Hyperglycemia-induced vasculopathy in the murine vitelline vasculature: correlation with PECAM-1/CD31 tyrosine phosphorylation state. Am J Pathol. 1999;154(5):1367-1379.

81. Evans PC, Taylor ER, Kilshaw PJ. Signaling through CD31 protects endothelial cells from apoptosis. Transplantation. 2001;71(3):457-460.

82. Thygesen K, Alpert JS, Jaffe AS, Chaitman BR, Bax JJ, Morrow DA, et al. Fourth universal definition of myocardial infarction (2018). J Am Coll Cardiol. 2018;72(18):2231-2264.

83. Eggers KM, Lindahl B. Application of cardiac troponin in cardiovascular diseases other than acute coronary syndrome. Clin Chem 2017;63(1):223-235.

84. Eggers KM, Bertil L. Application of cardiac troponin in cardiovascular diseases other than acute coronary syndrome. Clin Chem. 2017;63(1):223-235.

85. Heeschen C, Dimmeler S, Hamm CW, van den Brand MJ, Boersma E, Zeiher AM, et al. Soluble CD40 ligand in acute coronary syndromes. New Engl J Med. 2003;348(12):1104-1111.

86. Cipollone F, Ferri C, Desideri G, Paloscia L, Materazzo G, Mascellanti M, et al. Preprocedural level of soluble CD40L is predictive of enhanced inflammatory response and restenosis after coronary angioplasty. Circulation. 2003;108(22):2776-2782.

87. Schönbeck U, Varo N, Libby P, Buring J, Ridker PM. Soluble CD40L and cardiovascular risk in women. Circulation. 2001;104(19):2266-2268. 
88. Kong C, Gao RY, Yan XB, Huang LS, Qin HL. Probiotics improve gut microbiota dysbiosis in obese mice fed a high-fat or high-sucrose diet. Nutrition. 2019;60:175-184.

89. Li K, Zhang L, Xue J, Yang X, Dong X, Sha L, et al. Dietary inulin alleviates diverse stages of type 2 diabetes mellitus via anti-inflammation and modulating gut microbiota in $\mathrm{db} / \mathrm{db}$ mice. Food Funct. 2019;10(4):1915-1927.

90. Amabebe E, Anumba DOC. Female gut and genital tract microbiota-induced crosstalk and differential effects of short-chain fatty acids on immune sequelae. Frontiers Immunol. 2020;11:2184.

91. Franzosa EA, Sirota-Madi A, Avila-Pacheco J, Fornelos N, Haiser HJ, Reinker S, et al. Gut microbiome structure and metabolic activity in inflammatory bowel disease. Nat Microbiol. 2018;4(2):293-305.

92. Loader J, Roustit M, Baltzis D, Veves A. Vascular dysfunction, inflammation, and exercise in diabetes. Diabetes and Exercise. Humana Press, Cham 2018, 137-150.

93. Ren J, Sowers JR, Zhang Y. Metabolic stress, autophagy, and cardiovascular aging: from pathophysiology to therapeutics. Trends Endocrin Met. 2018;29(10):699-711.

94. Franco-Obregón A, Gilbert JA. The microbiome-mitochondrion connection: common ancestries, common mechanisms, common goals. Msystems. 2017;2(3): e00017-e00018.

95. Tang W, Kitai T, Hazen SL. Gut microbiota in cardiovascular health and disease. Circ Res. 2017;120(7): 1183-1196.

96. Jin L, Shi XM, Yang J, Zhao YY, Xue LX, Xu L, et al. Gut microbes in cardiovascular diseases and their potential therapeutic applications. Protein Cell. 2020;12(5):346-359.

\section{Figures}


A
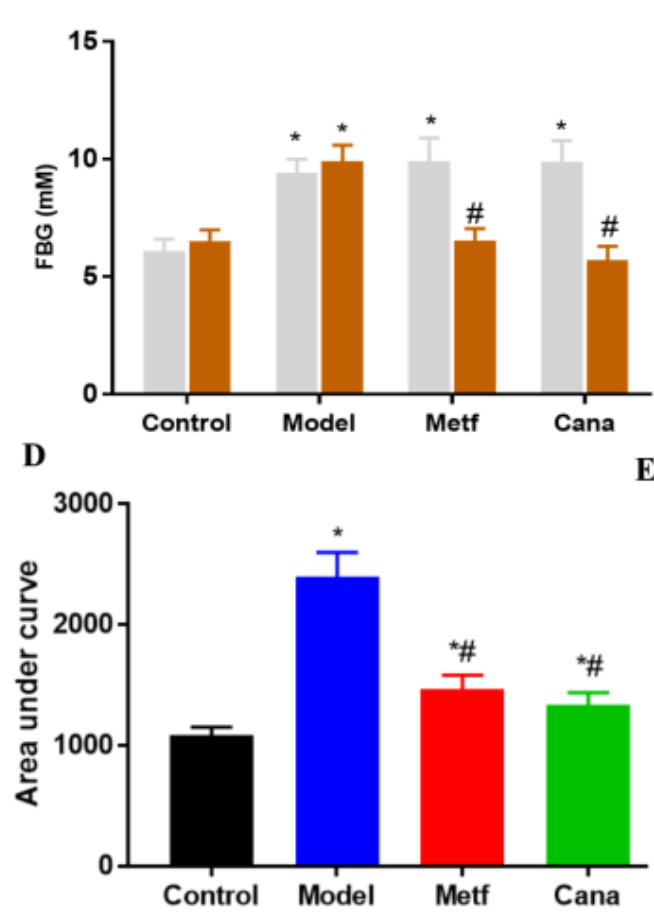

G

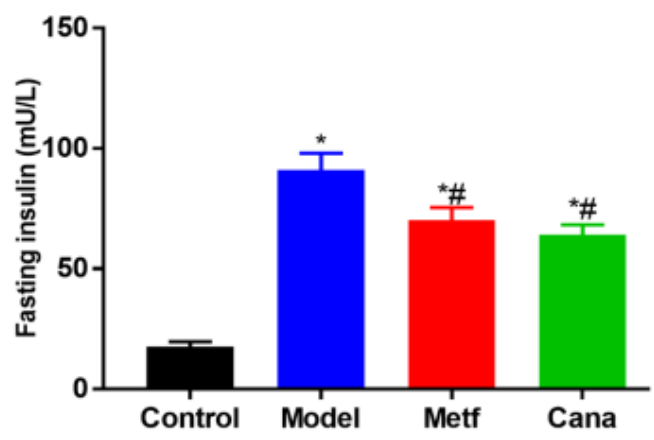

H

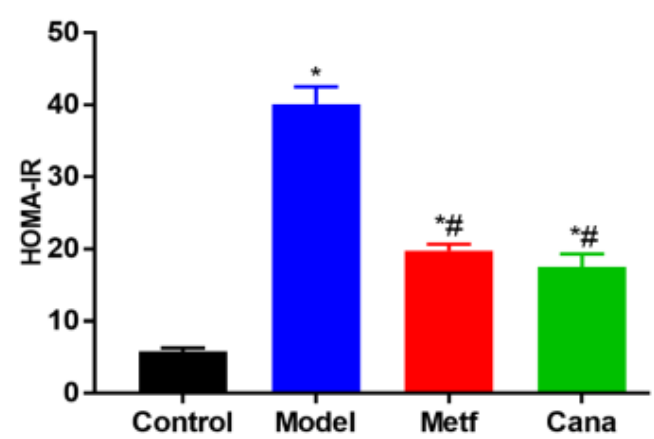

B

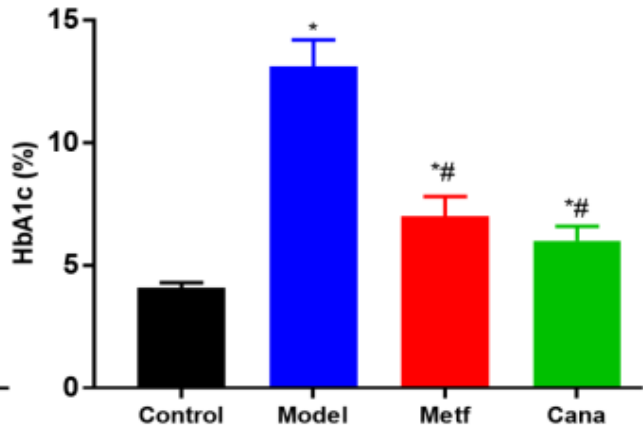

E

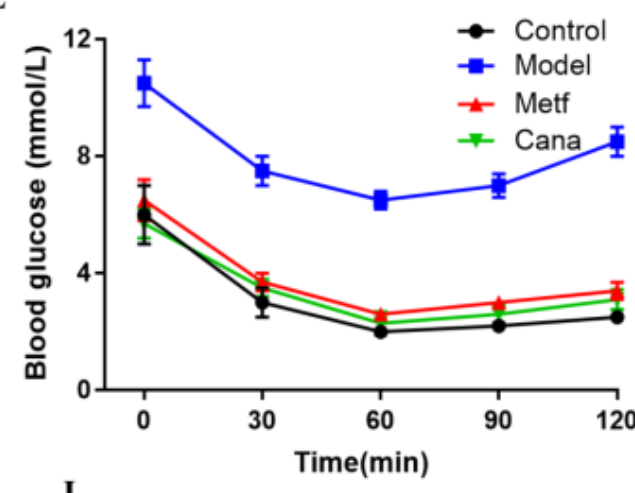

I
C
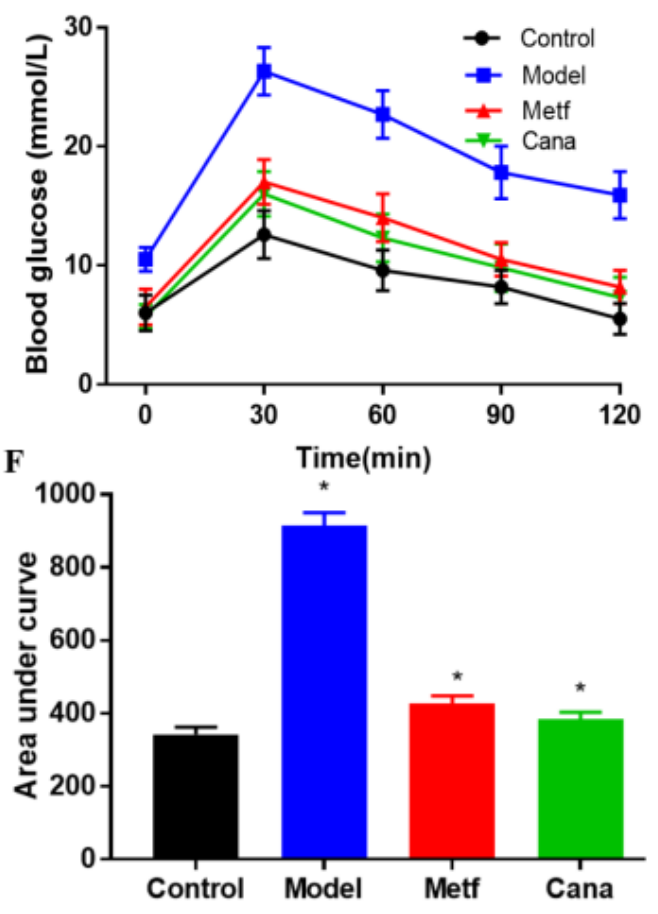

Glucagon

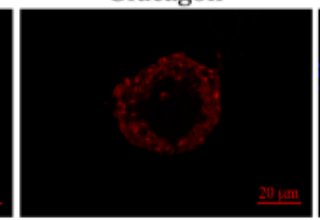

Nucleus

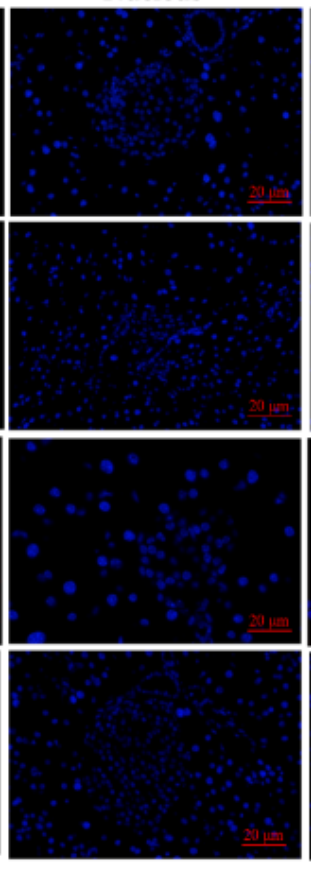
Merge

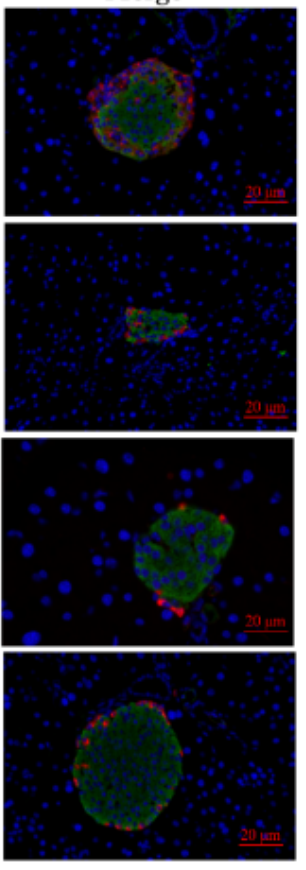

Figure 1

Cana administration improved glucose-insulin homeostasis in T2DM mice. Fasting blood glucose (A), grey and brown color represent the fasting blood glucose at the 24th week and at the end of the trial, respectively; HbA1c (B); Curve of OGTT (C) and areas under the curve (D) at the end of the trial; Curve of IPITT (E) and areas under the curve (F) at the end of the trial; Fasting insulin (G); HOMA-IR (H); Immunofluorescence staining for insulin (green), glucagon (red), and nucleus (blue) (I), scale bar: $20 \mu \mathrm{m}$. 
Data are expressed as mean \pm SEM. * $p<0.05$, compared with the Control group; \# $p<0.05$, compared with the Model group.

A

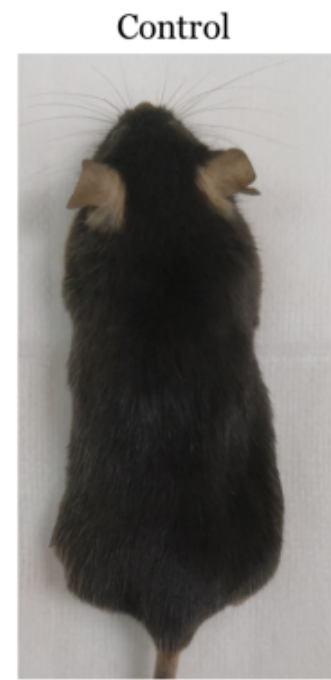

B

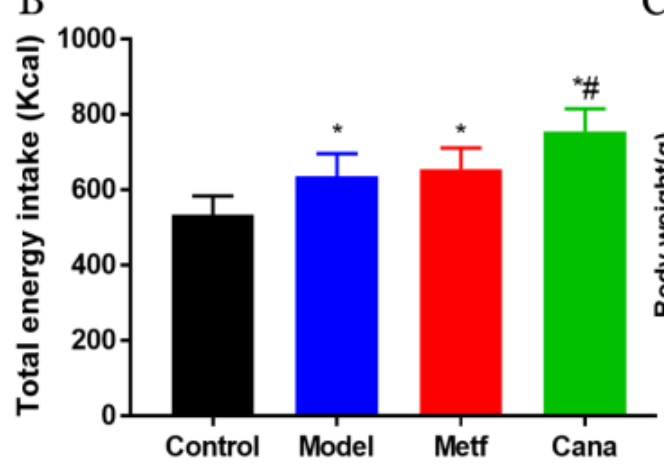

$\mathrm{E}$

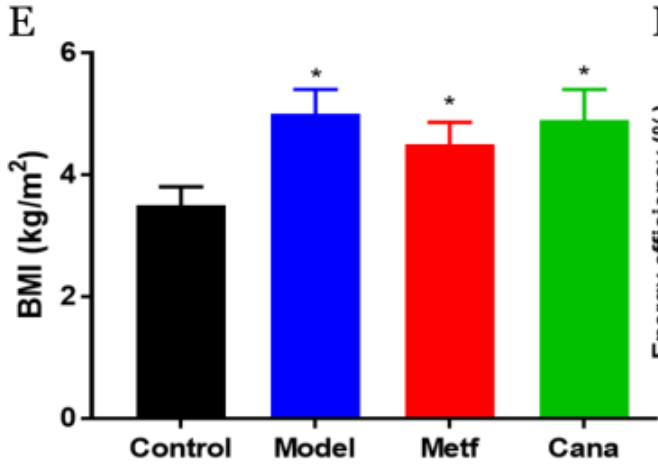

Model

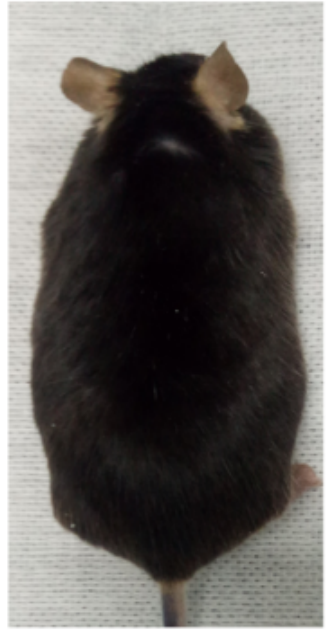

C
Metf
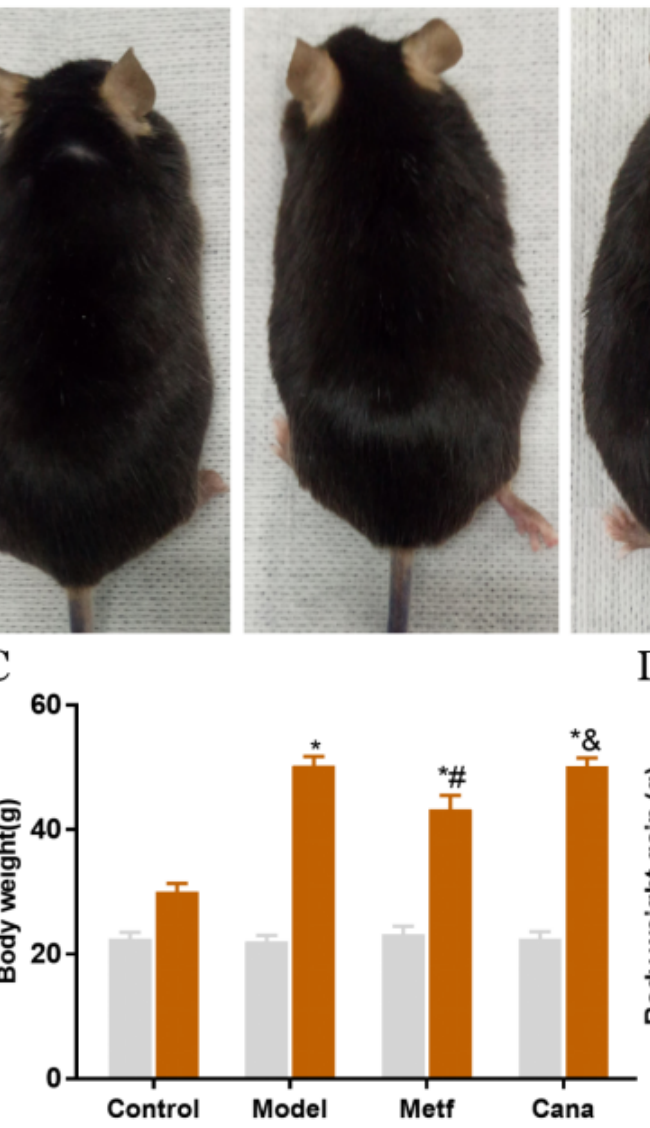

F
Cana

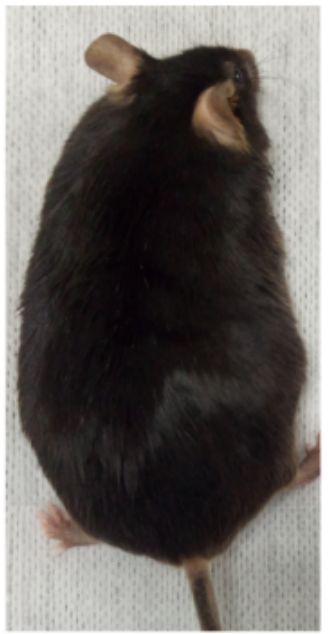

D
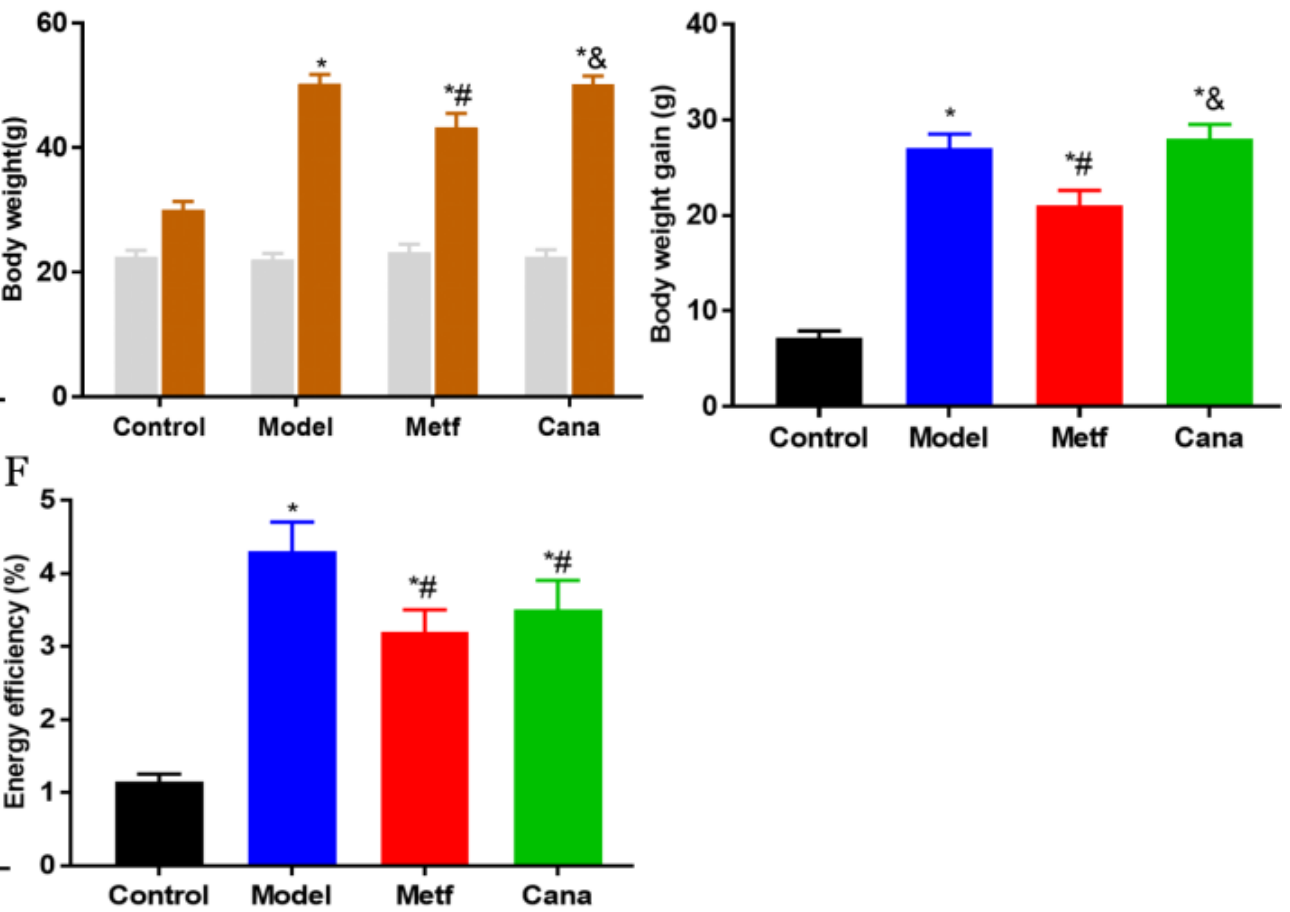

\section{Figure 2}

Effects of Cana treatment on physical parameters in T2DM mice. The gross appearance characteristics of mice with different treatments $(A)$; Total energy intake $(B)$; Body weight $(C)$, grey and brown color represent the body weight at the beginning and at the end of the trial, respectively; Body weight gain (D); BMI (E), calculated by body weight/height2; Energy efficiency (F), calculated by body weight gain/total energy intake. Data are expressed as mean \pm SEM. * $p<0.05$, compared with the Control group; \# $p<$ 0.05 , compared with the Model group; \& $<<0.05$, the Cana group compared with the Metf group. 

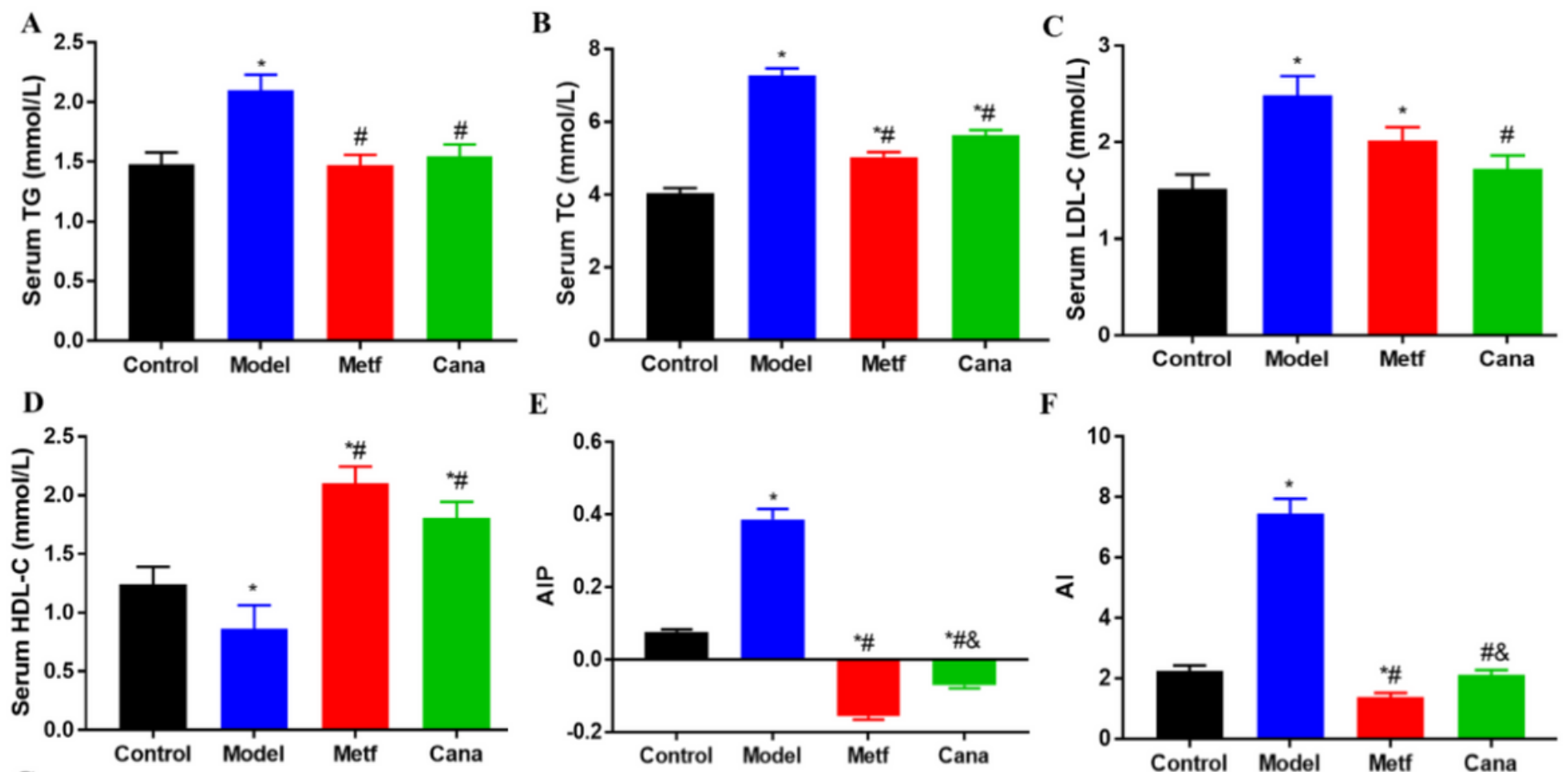

E

F
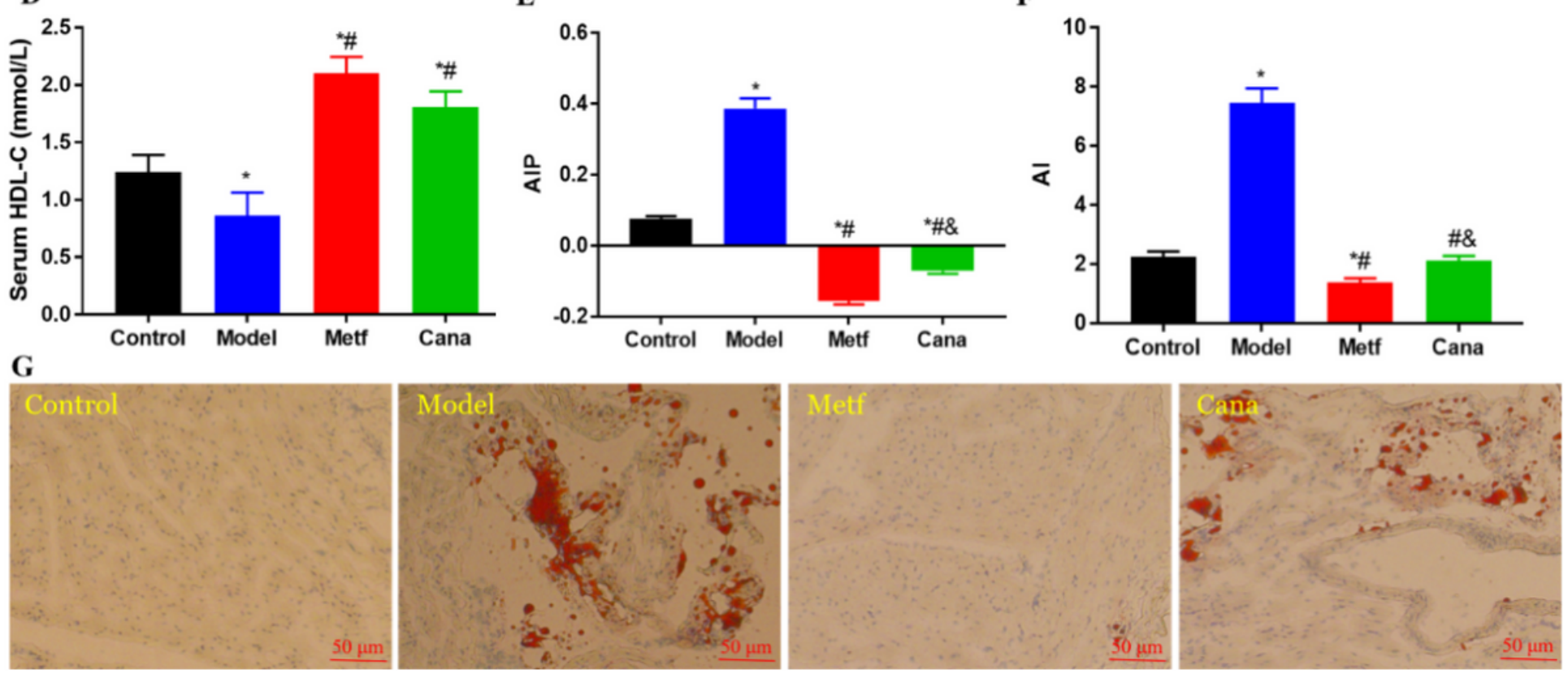

Figure 3

Effects of Cana on lipids profile in T2DM mice. Serum TG (A), TC (B), LDL-C (C), and HDL-C (D) levels; AIP (E), calculated by $\lg (\mathrm{TG} / \mathrm{HDL}-\mathrm{C}) ; \mathrm{Al}(\mathrm{F})$, calculated by (TC-HDL-C)/HDL-C; cardiac tissues with Oil red $\mathrm{O}$ staining (G), scale bar: $50 \mu \mathrm{m}$. Data are expressed as mean \pm SEM. ${ }^{*} p<0.05$, compared with the Control group; \# $p<0.05$, compared with the Model group; \& $p<0.05$, the Cana group compared with the Metf group. 


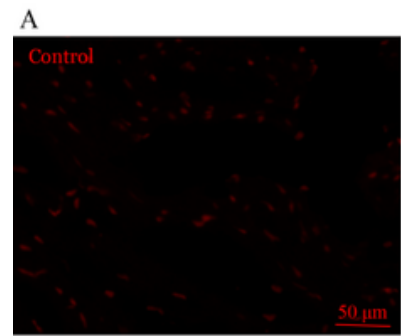

B

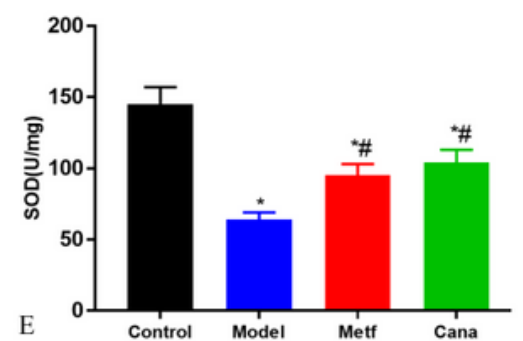

C

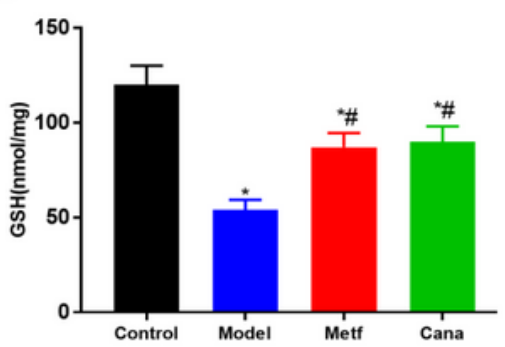

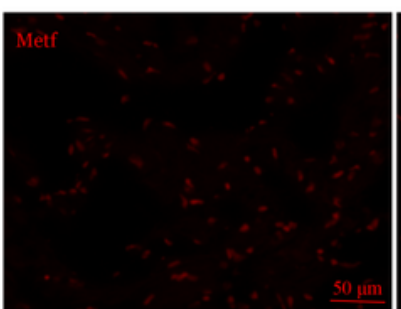

D

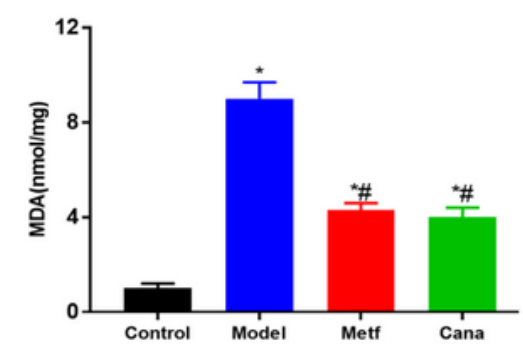

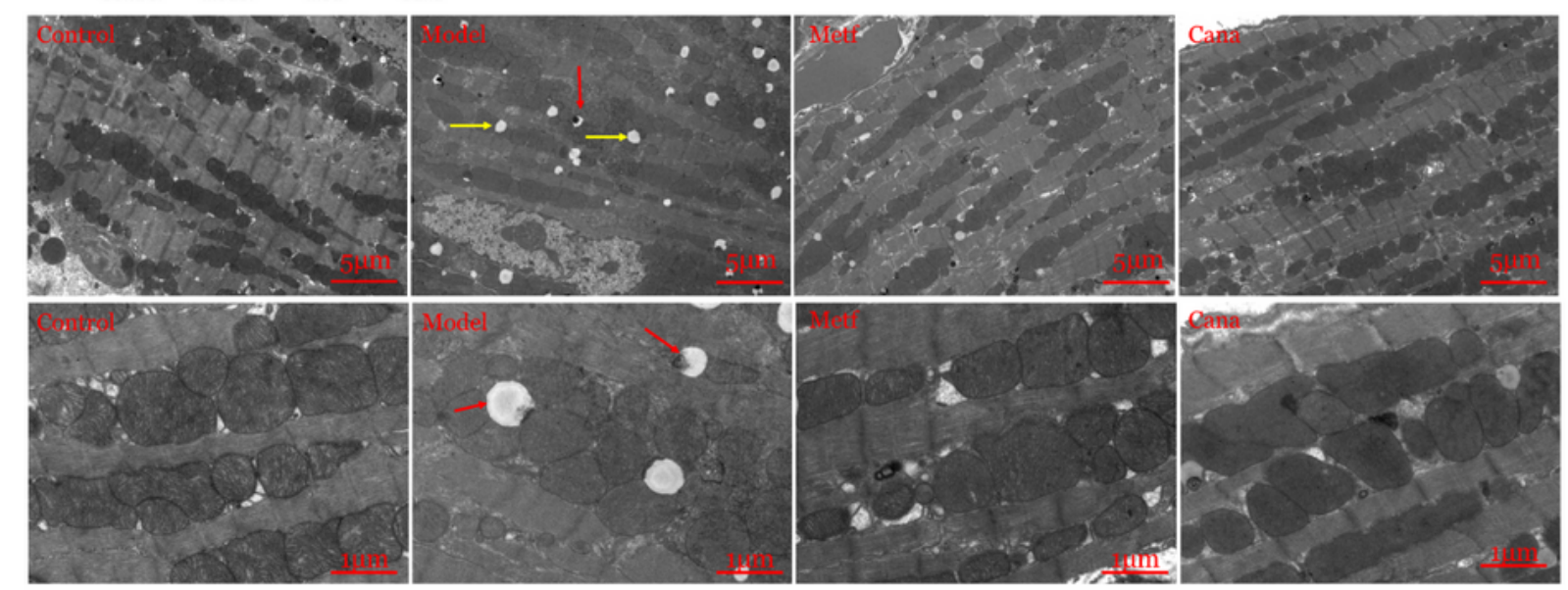

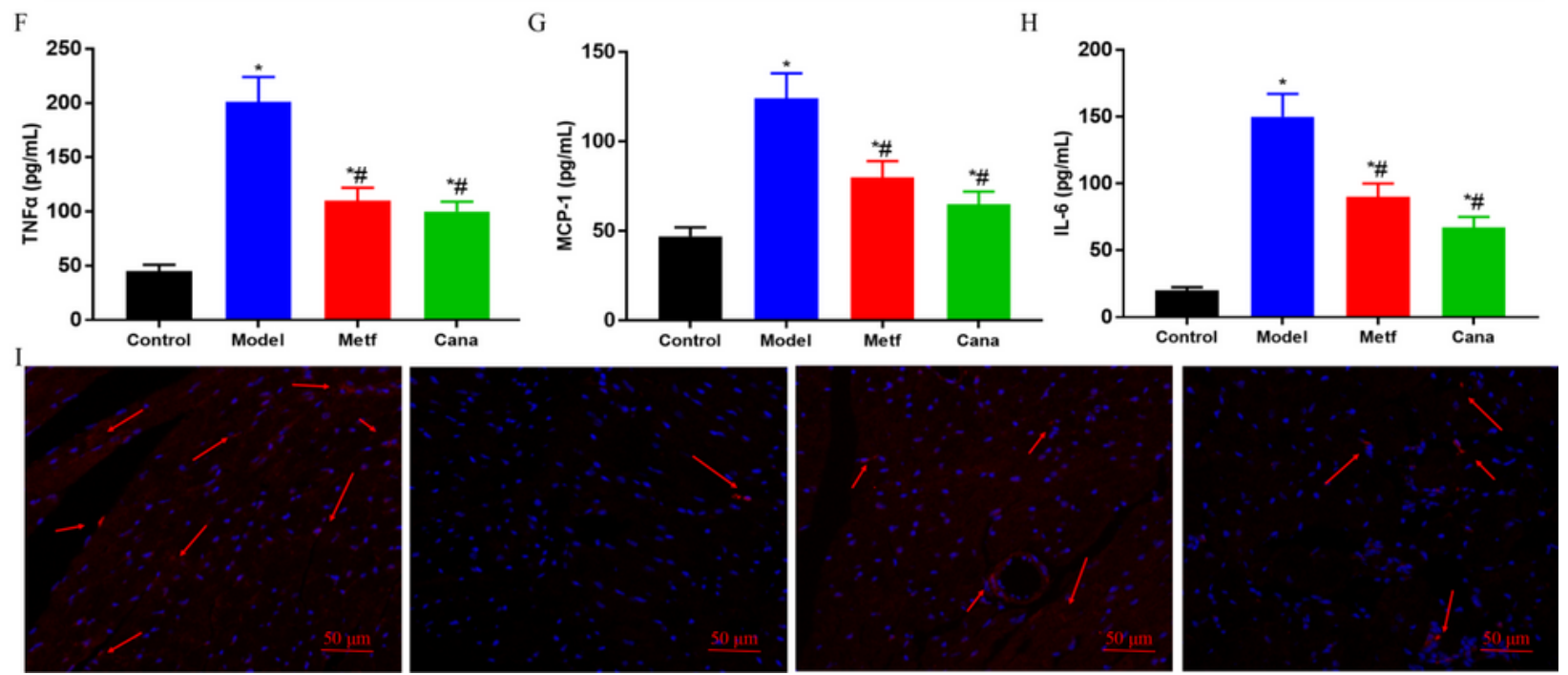

Figure 4

Effect of Cana on relieving oxidative stress and ameliorating systematic inflammation in T2DM mice. ROS levels in cardiac tissues (A), detected by in situ DHE staining, scale bar: $50 \mu \mathrm{m}$; SOD (B), GSH (C), and MDA (D) levels in cardiac tissues; The regulatory effect of Cana on cardiac mitochondria in myocardial tissues $(E)$. The scale bar is $5 \mu \mathrm{m}$ and $1 \mu \mathrm{m}$ in the upper panel and bottom panel, respectively; Cytokine concentrations of TNFa (F), MCP-1 (G), and IL-6 (H) in serum; The immunofluorescence staining 
of HO-1 in cardiac tissues, scale bar: $50 \mu \mathrm{m}(\mathrm{I})$. Data are expressed as mean $\pm \mathrm{SEM}$. ${ }^{*} \mathrm{p}<0.05$, compared with the Control group; \# $p<0.05$, compared with the Model group.

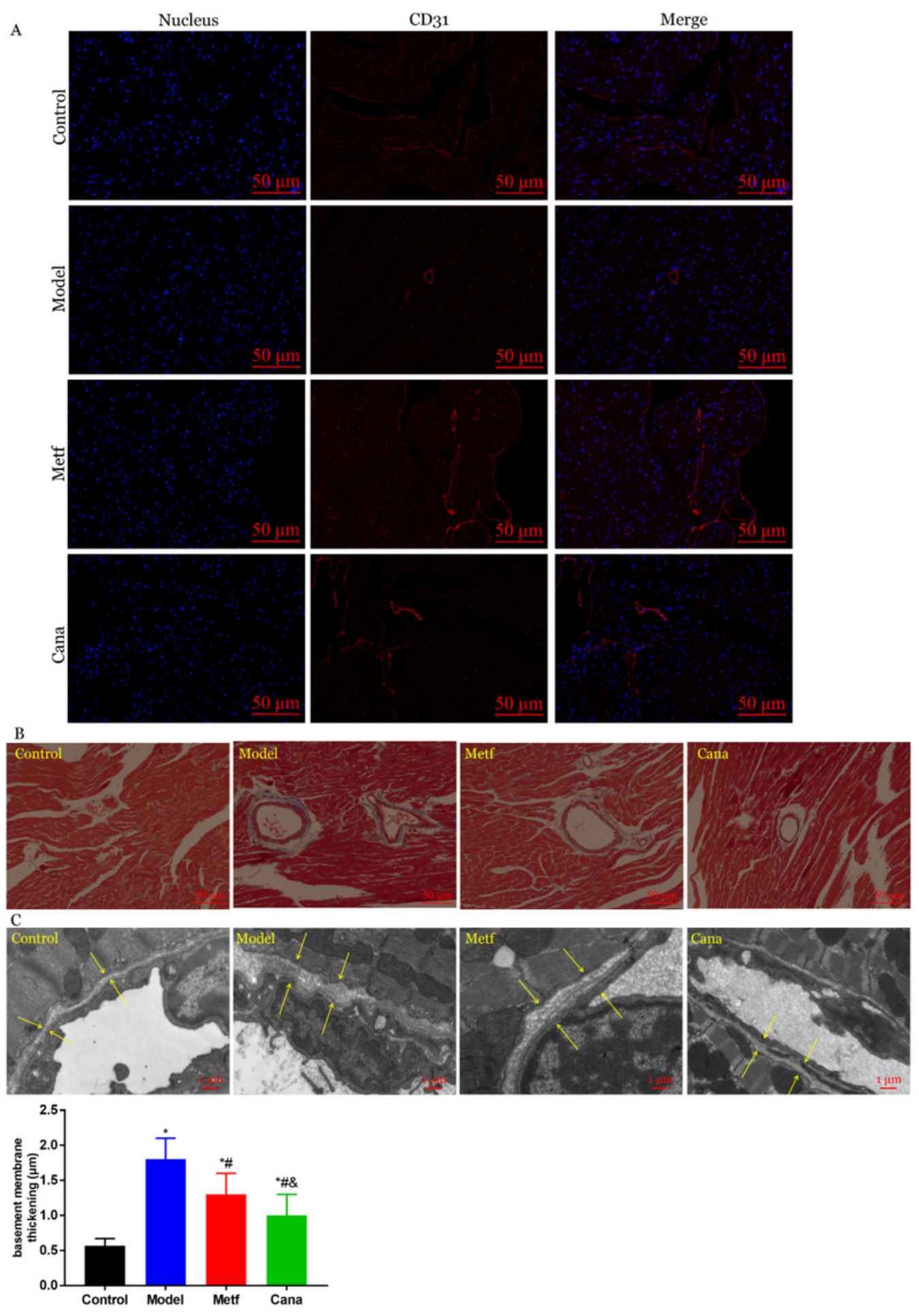

Figure 5

Effect of Cana on cardiovascular abnormalities in T2DM mice. The expression of CD31 in cardiac tissues (A), immunofluorescence staining for CD31 (red), and nucleus (blue), scale bar: $50 \mu \mathrm{m}$; Histological analysis of vascular fibrosis with Masson's staining (B), scale bar: $50 \mu \mathrm{m}$; Changes in vascular basement 
membrane observed by TEM (C), scale bar: $1 \mu \mathrm{m} .{ }^{*} p<0.05$, compared with the Control group; \# $p<0.05$, compared with the Model group; \& $p<0.05$, the Cana group compared with the Metf group.
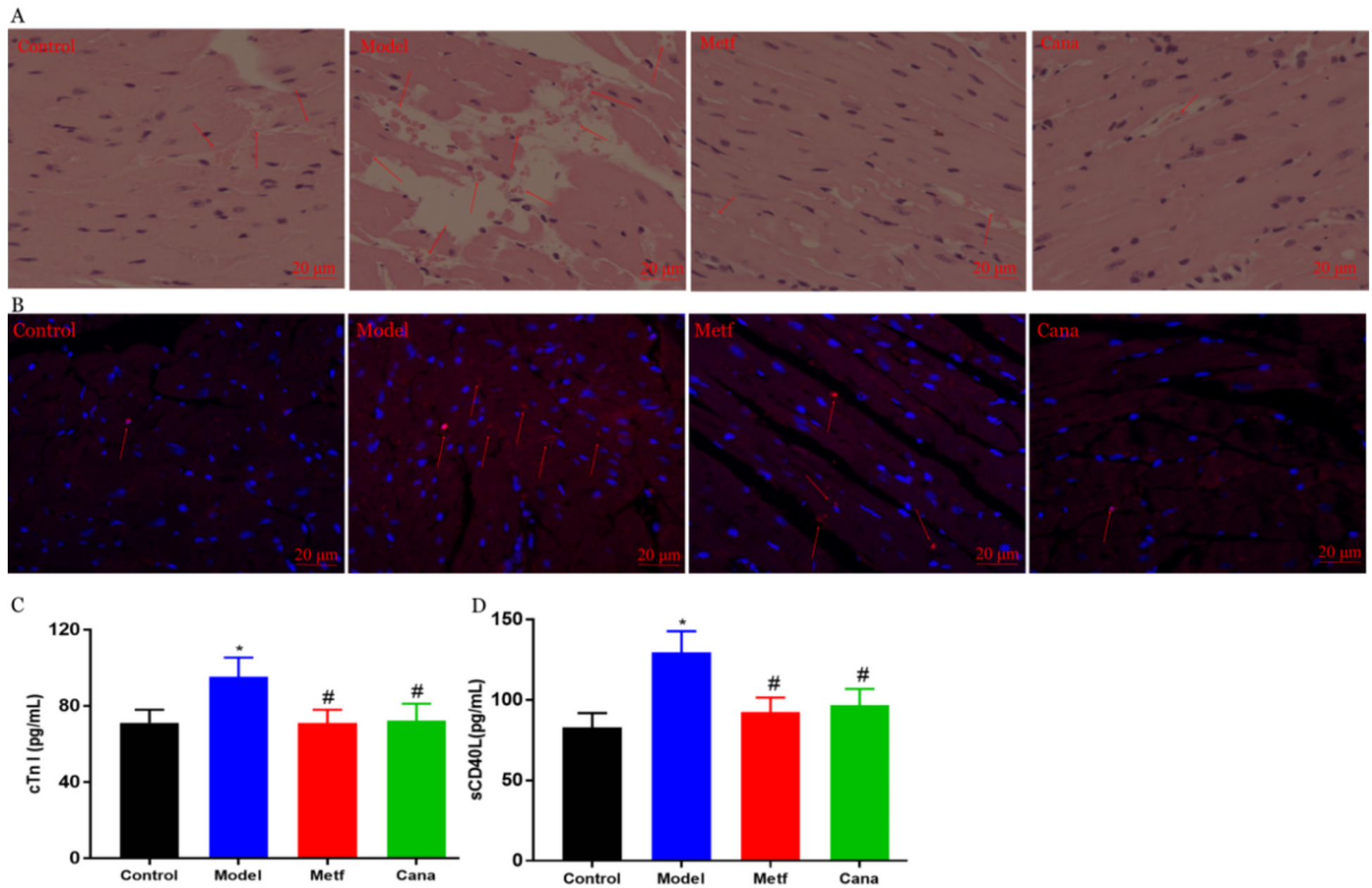

Figure 6

Effects of Cana on improving myocardial injury in T2DM mice. H\&E staining (A), scale bar: $20 \mu \mathrm{m}$; TUNEL assay to detect apoptosis in cardiac tissues (B), blue represents nucleus while red represents fragmented DNA, scale bar: $20 \mu \mathrm{m}$; Levels of cTn I (C) and sCD40L (D) in serum. ${ }^{*} p<0.05$, compared with the Control group; \# $p<0.05$, compared with the Model group; \& $p<0.05$, the Cana group compared with the Metf group. 

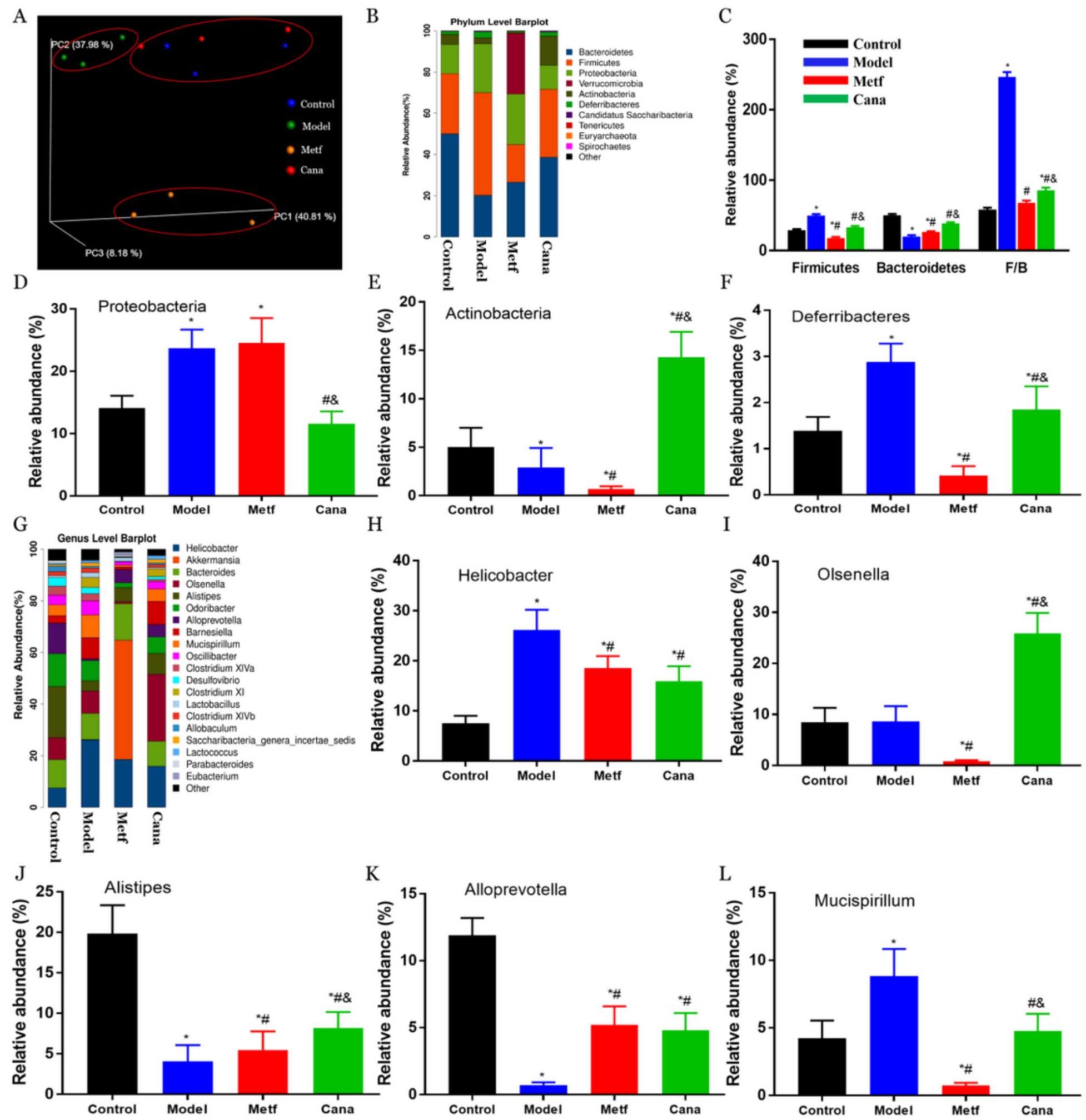

Figure 7

Structural modulation of colonic microbiota by Cana treatment in T2DM mice. PCoA plot of colonic microbiota (A); Relative abundance at the phylum level (B), including Firmicutes and Bacteroidetes (C), Proteobacteria (D), Actinobacteria (E), Deferribacteres (F) in different groups; Relative abundance at genus level (G), including Helicobacter $(\mathrm{H})$, Olsenella (I), Alistipes (J), Alloprevotella (K), and Mucispirillum 
(L). Data are expressed as mean \pm SEM. * $p<0.05$, compared with the Control group; $\# p<0.05$, compared with the Model group; \& $p<0.05$, the Cana group compared with the Metf group.

A

=

$\mathrm{C}$
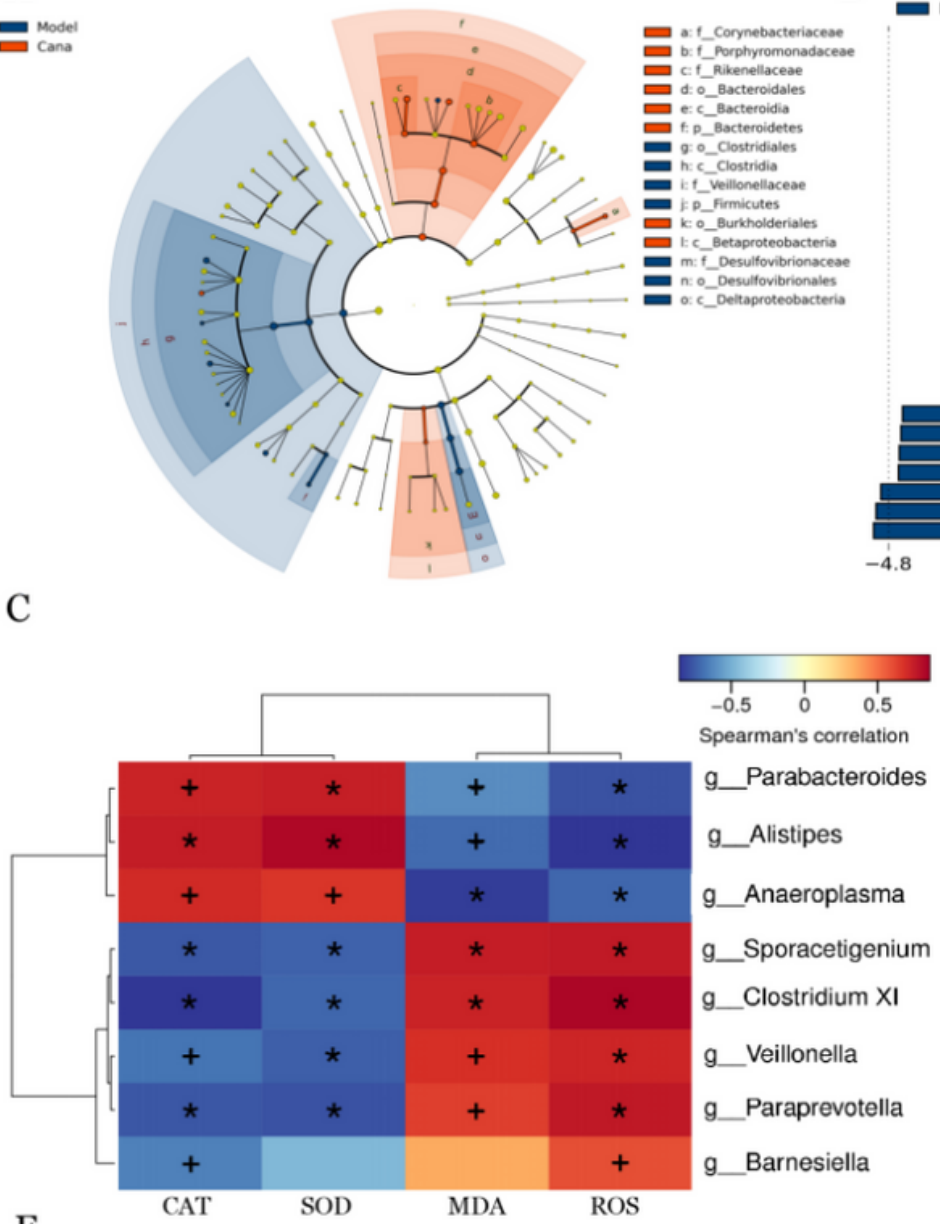

$\mathrm{E}$
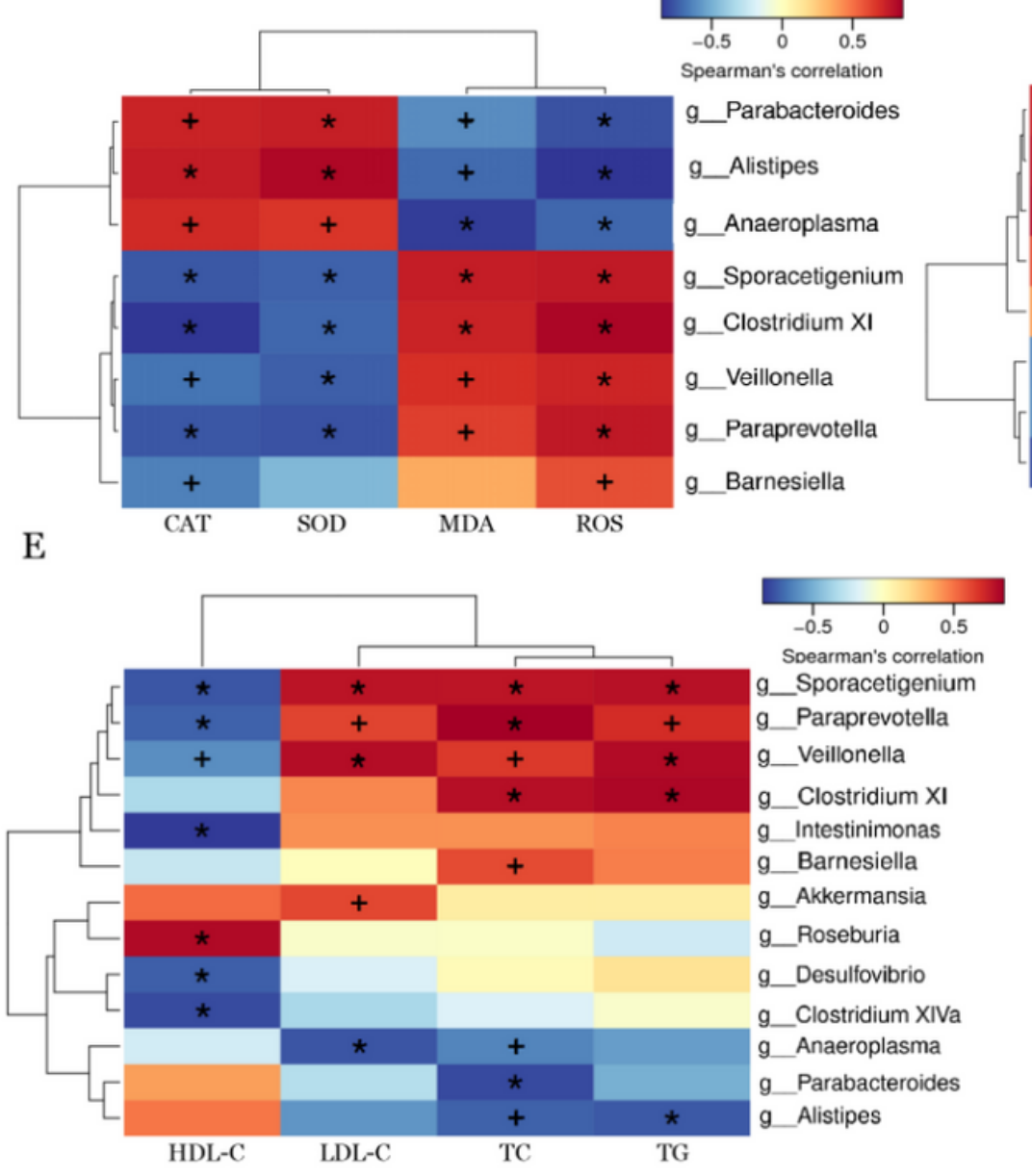

B

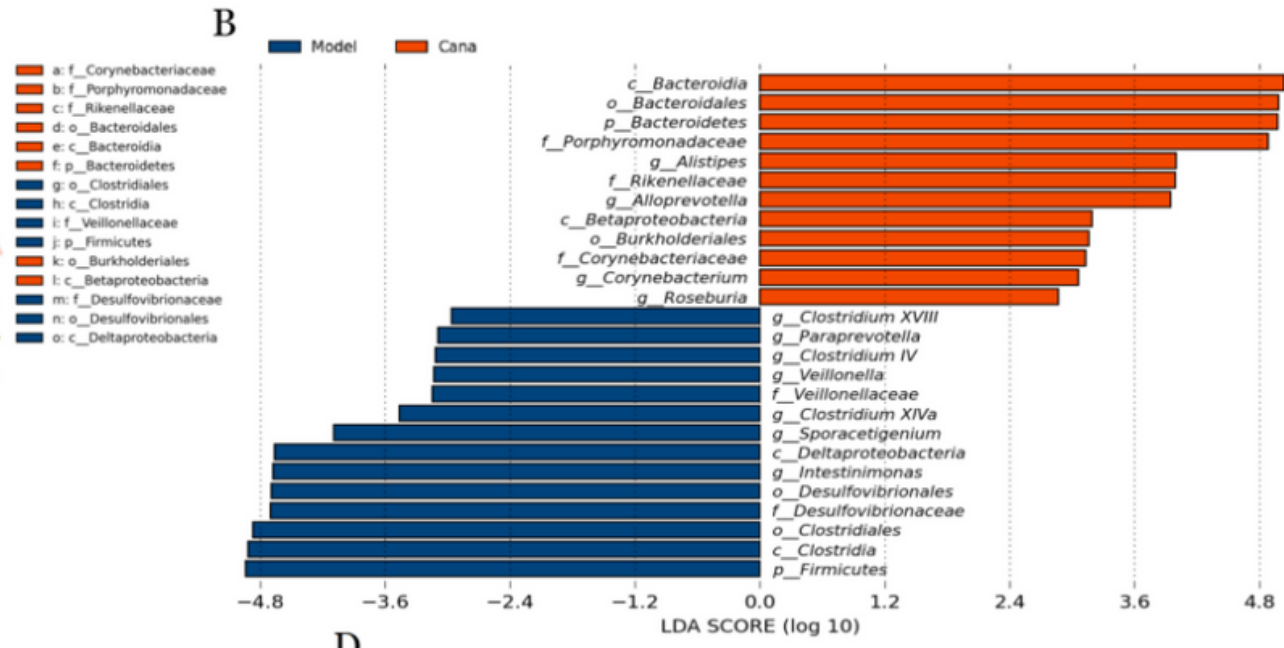

$\mathrm{D}$

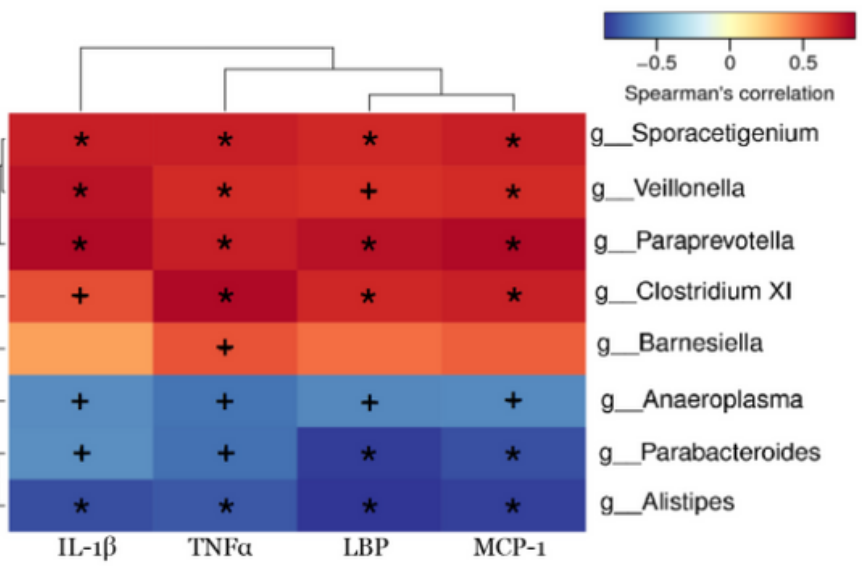

$\begin{array}{llll}\text { IL-1 } \beta & \text { TNFa } & \text { LBP } & \text { MCP-1 }\end{array}$

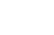


physiological indices and bacteria at genus level (C-E). The abscissa represents the physiological indices, and the ordinate represents the bacterial species. The shade of color directly indicates the correlation between physiological indices and bacteria. The correlation between microbial species and biomarker was visualized by color depth: the darker the color, the more relevant it is. $+: p<0.05, *: p<0.01$.

\section{Gut microbiota regulation}

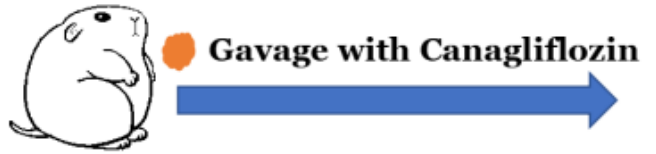

Mice with diabetic cardiovascular disease Hyperglycemia Insulin resistance Systemic inflammation Hematological parameters

\section{Cardiovascular protection}

Systemic inflammation Oxidative stress Hyperlipidemia Myocardial injury Cardiovascular abnormality Basement membrane thickness Lipid accuaccumulation

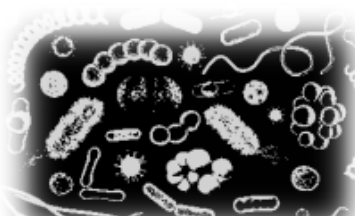

Firmicutes/Bacteroidetes Helicobacter Mucispirillum Alloprevotella Proteobacteria Roseburia

\section{Figure 9}

The possible mechanism of Cana on alleviating diabetic CVD.

\section{Supplementary Files}

This is a list of supplementary files associated with this preprint. Click to download.

- Supplementaldata.docx 